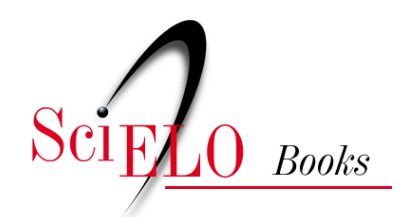

\title{
Capítulo II - Métodos de localización de fallas en sistemas eléctricos de distribución con presencia de generación distribuida
}

\author{
Ramón Pérez \\ Carmen Vásquez \\ Amelec Viloria
}

SciELO Books / SciELO Livros / SciELO Libros

PÉREZ, R., VÁSQUEZ, C., and VILORIA, A. Métodos de localización de fallas en sistemas eléctricos de distribución con presencia de generación distribuida. In: INGA ORTEGA, E., ed. Aplicaciones e innovación de la ingeniería en ciencia y tecnología [online]. Quito: Editorial AbyaYala, 2019, pp. 57-96. ISBN: 978-9978-10-491-0. https://doi.org/10.7476/9789978104910.0004.

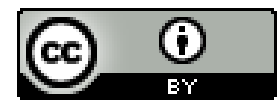

All the contents of this work, except where otherwise noted, is licensed under a Creative Commons Attribution 4.0 $\underline{\text { International license. }}$

Todo o conteúdo deste trabalho, exceto quando houver ressalva, é publicado sob a licença Creative Commons Atribição 4.0.

Todo el contenido de esta obra, excepto donde se indique lo contrario, está bajo licencia de la licencia $\underline{\text { Creative }}$ Commons Reconocimento 4.0 . 


\title{
Capítulo II
}

\section{Métodos de localización de fallas en sistemas eléctricos de distribución con presencia de generación distribuida}

\author{
Fault Location Methods in Electric \\ Distribution Systems with Presence of \\ Distributed Generation
}

\author{
Ramón Pérez* Carmen Vásquez§ Amelec Viloria† \\ *email: rperezp @ups.edu.ec \\ §email: cvasquez @unexpo.edu.ve \\ †email: avioloria7@cuc.edu.co
}

\section{Resumen}

La localización de fallas en los sistemas eléctricos, especialmente en los de distribución, es un problema que afecta no solo a los usuarios, sino también a las empresas prestadoras del servicio eléctrico; con mayor tiempo invertido en este período, se incrementa las pérdidas por la energía no facturada y las molestias en los usuarios, disminuyendo la calidad del servicio. Son distintas las causas por las cuales se incrementa este tiempo, entre éstas la confusión que pudiera existir en los sistemas de localización que actúan bajo la presencia de fuentes de generación distribuida en las redes de distribución. En este sentido, la presente investigación muestra una revisión de los métodos utilizados para la localización de fallas en sistemas eléctricos de distribución que tengan presencia de fuentes de generación distribuida. Las principales características de los métodos son estudiadas y sus más recientes aplicaciones para tratar este problema.

\section{Palabras clave}

Fallas, inteligencia artificial, generación distribuida, sistemas eléctricos de distribución. 


\begin{abstract}
The location of faults in the electrical systems, especially in distribution, is a problem that affects not only the users, but also the companies providing the electric service; with more time invested in this period, the losses due to the unbilled energy and the inconvenience in the users are increased, decreasing the quality of the service. The causes for which this time is increased are different, among them the confusion that could exist in the location systems that act under the presence of sources of distributed generation in the distribution networks. In this sense, the present investigation shows a revision of the methods used for the location of faults in electrical distribution systems that have presence of sources of distributed generation. The main characteristics of the methods are studied and their most recent applications to deal with this problem.
\end{abstract}

\title{
Keywords
}

Faults, artificial intelligence, distributed generation, electric distribution systems.

\section{Introducción}

Un Sistema Eléctrico de Distribución (SED) es un conjunto de instalaciones y equipos encargados de entregar la energía eléctrica a los distintos usuarios. En general, existen tres (3) tipos de configuraciones para este tipo de sistema y que se suelen llamar radial, anillo y malla, cada una con sus características, ventajas y desventajas. La configuración radial es más económica y sencilla de operar, es la más común para alimentar cargas de tipo residencial y no críticas [1]. Adicionalmente, según su construcción, estos sistemas se clasifican en aéreos y subterráneos, siendo los aéreos los más comunes [2].

Los SED no son inmunes a las interrupciones del servicio eléctrico ocasionadas por fallas. El tiempo de una interrupción depende de la detección de la falla por el dispositivo de protección, su apertura y despeje, de la localización de la falla y de la reparación necesaria para restablecer el servicio. La localización de la falla es el punto crítico del proceso y el principal inconveniente está relacionado con aspectos de infraestructura, es decir, la mayoría de los circuitos en los SED no disponen de localizadores de fallas, la configuración de los circuitos no es homogénea, ya que generalmente contienen diferentes secciones de conductor, longitudes y presentan múltiples derivaciones de cargas a lo largo de cada alimentador [3]. 
Actualmente, los SED están presentando penetración de Generación Distribuida (GD), la cual es universalmente aceptada como una solución efectiva y económica para responder a los incrementos en las demandas de energía del sistema, debido a que representa una mejor opción para corregir los problemas de pérdidas de energía, perfiles de tensión, problemas de cargabilidad de líneas, seguridad y confiabilidad, por mencionar algunos [4]. La integración de la GD a los SED convencionales modifica las amplitudes de las señales de falla (tensión y corriente) lo cual afecta significativamente la exactitud de los algoritmos de localización de fallas [5], por esta razón, este problema resulta de importancia tratarlo cuando existe presencia de GD en las redes [6].

Los métodos algorítmicos han sido utilizados para tratar este problema con la presencia de GD. Estos métodos dependen del modelo de la red y el error en la localización de la falla aumenta significativamente cuando se incrementa la potencia inyectada por las fuentes de GD a los SED [7]. Los autores de [8] utilizan el método basado en impedancia para localizar fallas en SED con presencia de GD. La técnica es validada en el circuito IEEE de 34 nodos considerando los diferentes tipos de falla que se puedan presentar con resistencias de falla entre 0 y $40 \Omega$ y penetración de GD entre 5 y $50 \%$. Los resultados los muestran errores de estimación menores al $2 \%$.

Los autores de [9] concluyen que los métodos basados en la impedancia están influenciados por la resistencia de falla y por la distancia entre la falla y el punto de medición. Mientras mayor sea la resistencia y la distancia de falla, mayor será el error en la estimación. En general, la exactitud de los métodos basados en la impedancia depende de los parámetros de la línea, sus características y del valor de carga. El error en la localización de estos métodos también se ve afectada por la complejidad de la red, como los sistemas no balanceados, múltiples laterales y resistencias de falla. Para fallas con múltiples estimaciones, el estado de los dispositivos de protección se utiliza comúnmente para identificar la ubicación real. Sin embargo, para un SED que no está equipado con el estado en línea de los dispositivos de protección, el problema de la múltiple estimación no podría resolverse [10]. Estos métodos proporcionan exactitud, pero con múltiple estimación en la localización de la falla [11]. 
En [12] localizaron fallas en un SED con presencia de GD utilizando Redes Neuronales Artificiales (ANN, por sus siglas en inglés) con el enfoque estándar de retropropagación. En este método, los datos de entrenamiento se basan en la corriente inyectada por cada fuente de fuente de GD durante la falla. Por lo tanto, la exactitud del método es altamente dependiente del número de fuentes de GD en el sistema. El principal inconveniente de las ANN es su alta dependencia de la cantidad y la calidad de los datos entrenados para producir un algoritmo bien entrenado. Una cantidad limitada de información o su inexactitud, afecta el desempeño del algoritmo para identificar correctamente la ubicación de la falla. Este problema ocurre en los SED con información limitada que resulta de un número insuficiente de dispositivos de monitoreo [10].

Las Máquinas de Soporte Vectorial (SVM, por sus siglas en inglés) han sido utilizadas para localizar fallas en SED con GD. Éstas se basan en patrones representados por medidas de tensión y corriente en la subestación y en las fuentes de GD. Esta técnica presenta mayor robustez cuando se incrementa el número de fuentes de GD en la red. En [13] presentan la aplicación de las SVM para diagnosticar fallas en SED con la presencia de GD. El enfoque propuesto se basa en las tres (3) tensiones y corrientes de fase que están disponibles en todas las fuentes, es decir, en la subestación y en los puntos de conexión de la GD. La metodología propuesta es ilustrada en un alimentador de distribución de la subestación de $132 / 11 \mathrm{kV}$ en la India con cargas en diferentes lugares y varias fuentes de GD. El esquema de localización de fallas propuesto es capaz de identificar con exactitud el tipo de falla, la ubicación de la sección del alimentador en falla y la impedancia de falla. El resultado de la simulación muestra el rendimiento satisfactorio en términos de clasificación y regresión. La exactitud de la clasificación para la línea en falla fue de 100, 99,95 y 92,06\%, con tres (3) fuentes de GD, dos (2) fuente de GD y una (1) fuente de GD, respectivamente. Para cualquier cambio en la topología del sistema, las SVM tiene que ser reentrenadas antes de su aplicación. Además, con un mayor número de fuentes de GD, el enfoque se hace más robusto. Diferentes esquemas de localización de fallas en SED han sido estudiados considerando los métodos tradicionales basados en el modelado matemático del sistema, en ondas viajeras de altas 
frecuencias, en inteligencia artificial y la integración de dos (2) o más de éstos, resultando en nuevas investigaciones que buscan dar solución a este problema. En este sentido, en esta investigación se presenta la revisión de los principales métodos para la localización de fallas en SED con presencia de GD. La descripción de los SED, la GD y las metodologías existentes para tratar este problema se presentan en las siguientes secciones, finalizando con las conclusiones.

\section{Sistemas eléctricos de distribución}

Los Sistemas Eléctricos de Potencia (SEP) y la prestación del servicio eléctrico incluyen las actividades de generación, transmisión, distribución, despacho y comercialización final de la energía. Entre estas actividades, la de distribución es conocida como el "gigante invisible" [2], por ser aproximadamente el 66\% de la inversión total del sistema, lo que implica que se debe realizar un trabajo riguroso a la hora de planificar, diseñar, construir, operar y mantenerlo. Su función primordial es llevar la energía eléctrica generada en las centrales a los usuarios finales y entregarla con los requisitos de calidad exigidos, es decir, de manera, segura, confiable y de adecuada prestación [14].

Los SED están constituidos por las subestaciones, conductores, transformadores y demás elementos de un sistema eléctrico que sirve para llevar la energía eléctrica desde la subestación distribuidora hasta los usuarios finales. Esta subestación incluye transformadores de potencia, barras, condensadores, seccionadores y reconectadores, entre otros elementos. Los alimentadores primarios de distribución, a la salida de las subestaciones, son trifásicos y operan a tensiones que van desde $1.000 \mathrm{~V}$ hasta $34.5 \mathrm{kV}$ y se encargan de distribuir la energía a los centros de consumo. A partir de estos centros se tienen sub-alimentadores ramales trifásicos y/o monofásicos. Los transformadores de distribución pueden ser de diferentes tipos y configuraciones y sus potencias nominales van desde 10 a $500 \mathrm{kVA}$ y suelen instalarse en los alimentadores primarios y sub-alimentadores para reducir el nivel de tensión de distribución en su circuito secundario al requerido por los usuarios [1]. Estos sistemas se clasifican de manera más general, en función de la disposición de sus 
conductores y equipos, en aéreos y subterráneos. En la Figura 1 se muestra un SED típico.

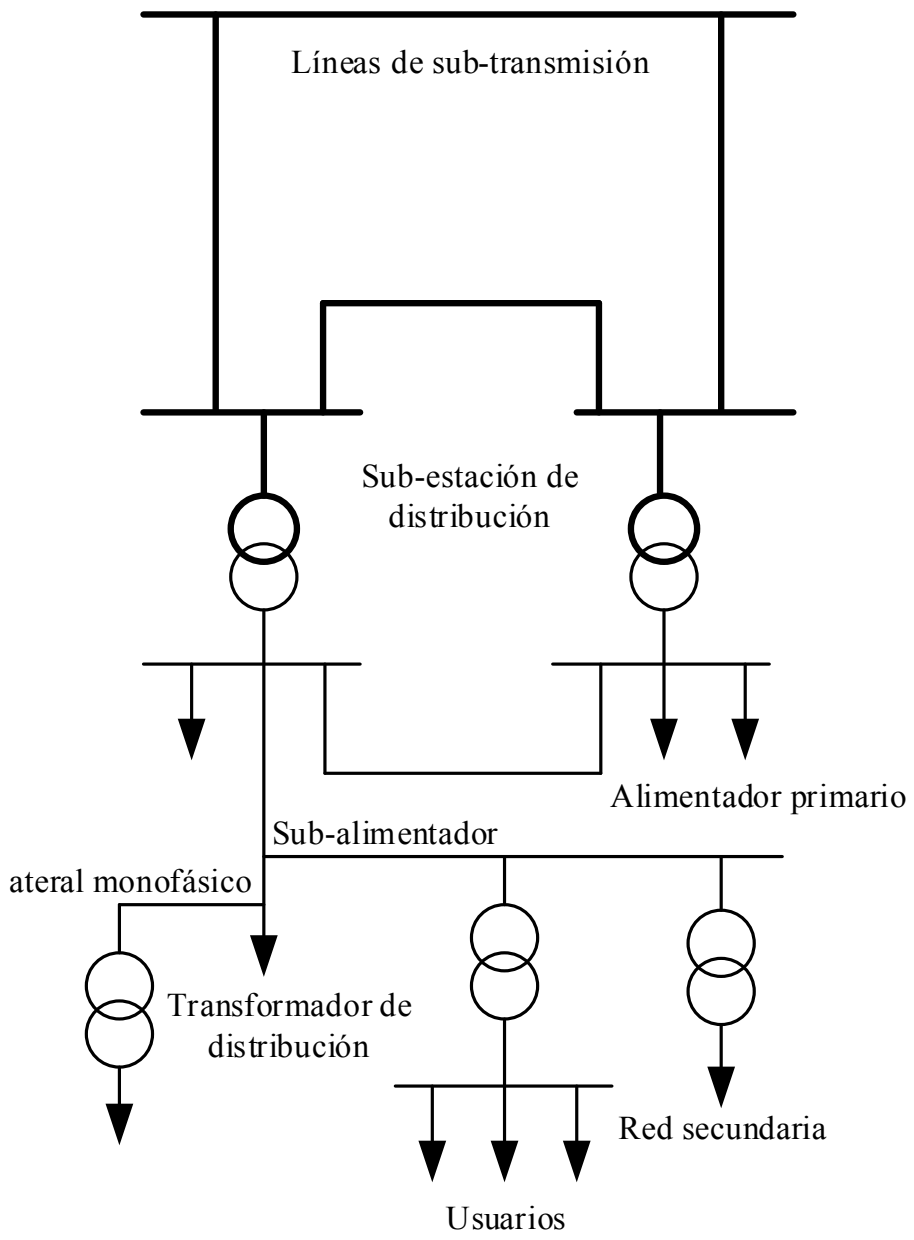

Figura 1. SED típico

Fuente: Adaptación de [15]

Según [2], las actividades de los SED están en constante evolución, por mencionar algunos de estos nos encontramos: 
- Expansión de las cargas.

- Normalización de los materiales, estructuras y montajes.

- Técnicas de diseño, análisis y operación.

- Métodos de trabajos específicos y normalizados.

- Programas de prevención de accidentes y de mantenimientos.

- Surgimiento de nuevas empresas de fabricación de equipos.

- Grandes volúmenes de información, datos y planos.

Los SED son de grandes longitudes y número de equipos, por lo que los estudios para garantizar la calidad de la energía eléctrica prestada a sus usuarios son complejos de realizar, porque se involucran un sinnúmero de variables.

\section{1) Interrupciones y las causas de fallas}

Las interrupciones del servicio eléctrico tienen una multitud de origen o causas, por lo que es necesario realizar una clasificación y análisis de éstas con el fin de minimizarlas. Una primera clasificación que se puede hacer es en base a su naturaleza, según ésta las interrupciones pueden ser programadas o forzadas [11].

Las interrupciones programadas son previstas, ya que con suficiente antelación se les informa a los usuarios afectados, para que puedan tomar las distintas previsiones que minimicen el impacto causado por la falta del suministro de energía eléctrica. Este tipo de interrupciones suelen ocurrir por labores de mantenimiento, ampliaciones o maniobras de la red.

Las interrupciones forzadas son consideradas más perjudiciales, por su carácter imprevisto, debido a que los usuarios no tienen oportunidad de tomar medidas que el caso requiera. Según [16], estas últimas se clasifican de manera general según su origen el cual puede ser:

- Origen desconocido: Incluye todas las interrupciones cuyas causas se desconocen, suelen ser transitorias y no dejan algún rastro.

- Origen climatológico: Incluye todas las interrupciones que tienen su origen en fenómenos climatológicos, tales como lluvias o descargas atmosféricas. 
- Origen externo: Incluye las interrupciones debidas a agentes externos al sistema eléctrico como son los animales (aves, roedores), árboles, ramas, vehículos, personas, por nombrar algunos.

- Origen interno: Incluye todas las interrupciones causadas por agentes o elementos pertenecientes al sistema eléctrico, entre los cuales destacan: falla en algún componente de la red, falla en los sistemas de protección, falla humana en el manejo del sistema, por nombrar algunos.

Según [17], otra forma de clasificar las interrupciones es de acuerdo con su duración, entre las cuales destacan:

- Momentáneas: Una interrupción momentánea se presenta cuando el valor eficaz de la tensión de suministro disminuye a un valor por debajo de 0,1 por unidad (pu) y durante un tiempo inferior a los tres ciclos (50 ms), pero superior o igual al medio ciclo $(8,33 \mathrm{~ms})$. No requiere la intervención humana para reparar y restablecer el normal funcionamiento del sistema

- Temporales: Una interrupción temporal se presenta cuando el valor eficaz de la tensión de suministro disminuye a valores por debajo de $0,1 \mathrm{pu}$, durante un tiempo inferior a un (1) min, pero superior o igual a los tres ciclos (50 ms). Tampoco requiere la intervención humana para reparar y restablecer el normal funcionamiento del sistema

- Permanentes o Sostenidas: Una interrupción permanente del servicio se origina cuando el valor eficaz de la tensión en el punto de suministro disminuye por debajo de 0.1 pu durante un tiempo superior a un (1) min. Este tipo de interrupción requiere la intervención humana para localizar, reparar y restablecer el normal funcionamiento del sistema y son las que mayor afectan la continuidad del servicio eléctrico.

Existe una clasificación más general para las interrupciones, que según su tiempo de duración pueden ser de corta o larga duración, en función de que la misma sea menor o mayor a un (1) minuto respectivamente. La tabla 1 muestra los requisitos establecidos por países europeos para establecer los límites de calidad. Adicionalmente, la tabla 2 muestra los datos de frecuencia y tiempo de duración promedio de las interrupciones de países de la misma región. 
Tabla 1. Requisitos de las interrupciones en diferentes países europeos

\begin{tabular}{|c|c|c|c|c|}
\hline País & $\begin{array}{c}\text { Duración por } \\
\text { evento (hr/evento) }\end{array}$ & $\begin{array}{l}\text { Indisponibilidad } \\
\text { (hr/año) }\end{array}$ & $\begin{array}{c}\text { Número de } \\
\text { eventos de larga } \\
\text { duración }\end{array}$ & $\begin{array}{c}\text { Número de } \\
\text { eventos de corta } \\
\text { duración }\end{array}$ \\
\hline Bélgica & 4 & & & \\
\hline \multirow{2}{*}{ Finlandia } & \multirow{2}{*}{12} & & $12-24(\mathrm{BT})$ & \\
\hline & & & 8-20 (MT) & \\
\hline Francia & 6 & & $2-6(\mathrm{AT})$ & 2-30 (MT) \\
\hline \multirow{2}{*}{ Italia } & \multirow{2}{*}{24} & & $2-6(\mathrm{BT})$ & \\
\hline & & & $3-5(\mathrm{MT})$ & \\
\hline Lituania & & $60(\mathrm{BT})$ & $1(\mathrm{AT})$ & \\
\hline Polonia & & & & \\
\hline \multirow{3}{*}{ Portugal } & \multirow{3}{*}{$20-24$} & $6-20(\mathrm{BT})$ & & \\
\hline & & 4-16 (MT) & & \\
\hline & & $4(\mathrm{AT})$ & & \\
\hline \multirow{3}{*}{ España } & \multirow{3}{*}{$18(\mathrm{BT})$} & 6-20 (BT) & & \\
\hline & & 4-16 (MT) & & \\
\hline & & $6(\mathrm{AT})$ & & \\
\hline Gran Bretaña & 18 (MT) & & & \\
\hline República Checa & 12 & & & \\
\hline Hungría & $12-18$ & & & \\
\hline
\end{tabular}

Tabla 2. Promedios de interrupciones del servicio eléctrico en países europeos durante el período 1996-2002

\begin{tabular}{|c|c|c|}
\hline País & $\begin{array}{c}\text { Frecuencia de la interrupción } \\
\text { (evento por año) }\end{array}$ & $\begin{array}{c}\text { Tiempo de Indisponibilidad del } \\
\text { servicio eléctrico (min/año) }\end{array}$ \\
\hline Australia & 0,59 & 35,23 \\
\hline Bélgica & 0,90 & 42,25 \\
\hline Finlandia & 4,06 & 186,67 \\
\hline Francia & 1,21 & 53,33 \\
\hline Alemania & 0,27 & 57,00 \\
\hline Gran Bretaña & 0,77 & 70,09 \\
\hline Irlanda & 1,34 & 235,67 \\
\hline Italia & 3,83 & 202,85 \\
\hline Noruega & 2,73 & 218,00 \\
\hline España & 2,98 & 153,00 \\
\hline Suecia & 2,07 & 99,00 \\
\hline Países Bajos & 0,38 & 27,50 \\
\hline
\end{tabular}


Hay diversas causas de fallas en los SED, según estudios llevados a cabo por el Instituto de Investigación de Potencia Eléctrica (EPRI, por sus siglas en inglés) aproximadamente el $40 \%$ de fallas ocurren durante períodos de condiciones climáticas adversas que incluyen la lluvia, la nieve y el hielo [18].

\section{Generación distribuida}

Según [19], GD significa generadores de pequeña capacidad de potencia conectados directamente a los SED; su propósito es proporcionar una fuente de energía eléctrica activa [20]. Ésta es considerada como una opción importante para solucionar la mayoría de problemas en los SED, tales como pérdidas en las líneas, perfiles de tensión, cargabilidad de las líneas, confiabilidad y también alcanzar el objetivo de utilizar recursos energéticos verdes y renovables [4].

La capacidad máxima de GD que puede conectarse a un SED depende de su potencia, la cual está correlacionada con su nivel de tensión. El diseño técnico de cada SED es único, por lo tanto, no se puede dar una definición general de la capacidad máxima que se puede conectar, sin embargo, de acuerdo con el rango de potencia de la GD se sugieren algunas clasificaciones, tal como se muestra en la Tabla 3.

Tabla 3. Clasificación de la GD de acuerdo con su rango de potencia

\begin{tabular}{|c|c|}
\hline Clasificación de la GD & Rango de potencia \\
\hline Micro & $\sim 1 \mathrm{~W}<5 \mathrm{~kW}$ \\
\hline Pequeña & $5 \mathrm{~kW}<5 \mathrm{MW}$ \\
\hline Mediana & $5 \mathrm{MW}<50 \mathrm{MW}$ \\
\hline Grande & $50 \mathrm{MW}<300 \mathrm{MW}$ \\
\hline
\end{tabular}

Fuente: [20]

De acuerdo con el tipo de tecnología disponible, la GD varía ampliamente, muchas de las tecnologías utilizan recursos de energía renovable. Estos se definen como recursos que generalmente no están expuestos al agotamiento, como la luz solar, la fuerza del viento, la materia orgánica (biomasa) y la del océano, por mencionar algunos. Otro tipo de fuentes de GD de acuerdo con su tecnología, 
son las provenientes de la producción combinada de calor y electricidad. Las turbinas de gas de ciclo combinado, motores de combustión interna, turbinas de combustión y pilas de combustible son parte de este ciclo combinado. En la Tabla 4 se muestra los valores típicos de potencia de GD de acuerdo con su tecnología.

Tabla 4. Valores típicos de potencia de GD de acuerdo con su tecnología

\begin{tabular}{|c|r|}
\hline Tecnología & \multicolumn{1}{|c|}{ Valor típico de potencia } \\
\hline Turbina de gas de ciclo combinado & $35-400 \mathrm{MW}$ \\
\hline Motores de combustión interna & $5 \mathrm{~kW}-10 \mathrm{MW}$ \\
\hline Turbinas de combustión & $1-250 \mathrm{MW}$ \\
\hline Micro turbinas & $35 \mathrm{~kW}-1 \mathrm{MW}$ \\
\hline \multicolumn{2}{|c|}{ Renovables } \\
\hline Micro hidroeléctricas & $25 \mathrm{~kW}-1 \mathrm{MW}$ \\
\hline Pequeñas hidroeléctricas & $1-100 \mathrm{MW}$ \\
\hline Turbina eólica & $200 \mathrm{~W}-3 \mathrm{MW}$ \\
\hline Arreglos fotovoltaicos & $20 \mathrm{~W}-100 \mathrm{~kW}$ \\
\hline Solar térmica & $1-10 \mathrm{MW}$ \\
\hline Biomasa basada en gasificación & $100 \mathrm{~kW}-200 \mathrm{MW}$ \\
\hline Pilas de combustible, fosfato & $200 \mathrm{~kW}-2 \mathrm{MW}$ \\
\hline Pilas de combustible, carbonato fundido & $250 \mathrm{~kW}-2 \mathrm{MW}$ \\
\hline Geotérmica & $5-100 \mathrm{MW}$ \\
\hline Energía del océano & $100 \mathrm{~kW}-1 \mathrm{MW}$ \\
\hline Baterías de almacenamiento & $500 \mathrm{~kW}-5 \mathrm{MW}$ \\
\hline
\end{tabular}

Los SED se diseñan generalmente en un esquema radial, que tradicionalmente se alimenta desde la subestación ubicada aguas arriba en el inicio del circuito. Por lo tanto, se tiene un flujo de potencia unidireccional y una misma corriente dirigida desde la subestación hacia el alimentador aguas abajo, lo que facilita el cálculo de corriente de falla. Por otro lado, el nivel de tensión de las barras disminuye en la medida en que se encuentren más alejadas de la subestación, por esta razón, tienen un bajo perfil de tensión que debe ser corregido por bancos de condensadores, compensadores síncronos, Sistemas Flexibles de Transmisión en Corriente Alterna (FACTS, por sus siglas en inglés) o fuentes de GD en estos puntos débiles. Aunque la adición de GD en los SED mejora el perfil de 
tensión, cambia drásticamente las características del sistema, particularmente en cuanto a la condición de falla y la respuesta de los dispositivos de protección, influyendo en los niveles de corriente y en la contribución de las fuentes [4].

\section{Revisión sobre los métodos de localización de fallas}

Los aspectos mayormente considerados por los métodos de localización de fallas en los SED están directamente relacionados con sus características, tales como heterogeneidad de los alimentadores (debido a los diversos tamaños y longitudes de los conductores), desbalances (debido a la presencia de cargas monofásicas y/o trifásicas) y la presencia de laterales a lo largo del alimentador principal, por mencionar algunos. Estos aspectos pueden introducir errores en la estimación de la localización de la falla si se usan modelos simplificados [11].

Los métodos de localización de fallas en SED pueden dividirse en tres (3) categorías, según [21]:

1. Métodos basados en la inspección visual.

2. Métodos convencionales.

3. Métodos basados en Inteligencia Artificial.

\section{1) Métodos basados en la inspección visual}

Inicialmente la localización de fallas en SED se realizaba de manera visual. Los dispositivos de protección en las subestaciones o el reclamo realizado por los usuarios del servicio a causa de una suspensión alertaban a cuadrillas de personal especializado a localizar las fallas de manera visual, para poder repararla y restablecer nuevamente el servicio. El tiempo total de indisponibilidad del servicio era función de la alerta de la falla, su localización y del tiempo utilizado para la reparación y restablecimiento, el cual podría variar de unos cuantos minutos a horas y días, dependiendo de diversos factores, entre estos, tipo de configuración (aérea o subterránea), de circuito (urbano o rural), de causa de la falla (agentes externos o internos al sistema), entre otros. 
Los requerimientos de la calidad en la prestación del servicio hicieron que fuera cada vez más exigente reducir tiempos de indisponibilidad del servicio y para poder llevar un control de los mismos comenzaron a aparecer los indicadores, según las regulaciones establecidas [22], [23]. La norma IEEE Std 1366 [24] incluye una variedad de indicadores en función del número de clientes o usuarios afectados, la potencia interrumpida o la cantidad de energía dejada de suministrar, relativo a cuantificar los efectos relacionados a la suspensión del suministro eléctrico. Las iniciativas para reducir los tiempos de indisponibilidad del sistema de manera diferente a la inspección visual comenzaron a aparecer, como se presentan a continuación.

\section{2) Métodos convencionales}

Estos incluyen a los métodos basados en la impedancia y en ondas viajeras [21]

\subsection{Métodos basados en la impedancia}

Según [25], los métodos basados en la impedancia usan las mediciones de tensión y corriente de la frecuencia fundamental disponibles en la subestación e información como topología del SED, datos de líneas y cargas para encontrar la localización de la falla. A diferencia de las líneas de transmisión, los alimentadores de distribución suelen tener cargas intermedias significativas, mientras que las mediciones de tensión y corriente sólo están disponibles en la subestación y/o en la fuente de GD. Por lo tanto, estos métodos inician el proceso de localización de fallas desde la primera sección de línea y resuelven iterativamente las ecuaciones que describen la condición de estado de falla para todas las secciones, una por una, para hacer una estimación de la distancia. En la Figura 2 se ilustra este método. 


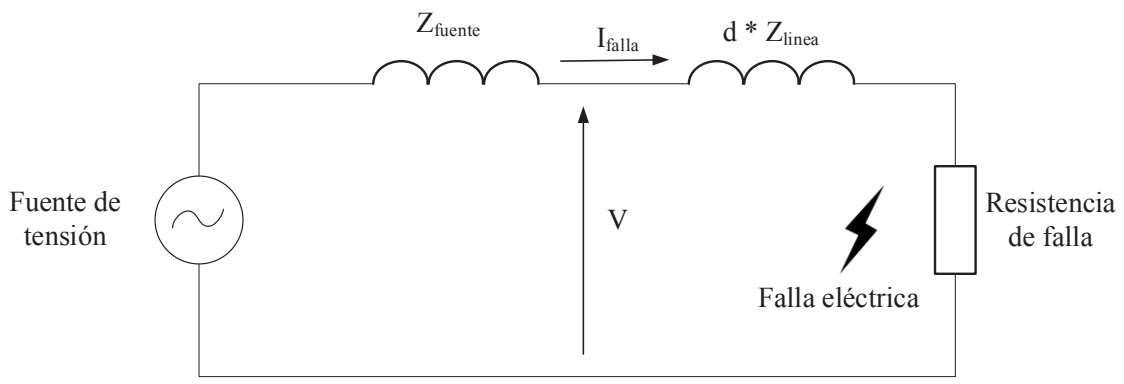

d: distancia de falla

Figura 2. Método de localización de falla basado en la impedancia

La formulación de la distancia de ocurrencia de la falla se puede expresar por medio de la ecuación (1):

$$
d=\frac{V}{I * Z_{l}}
$$

donde:

$V$ : Tensión durante la falla en $\mathrm{V}$.

I: Corriente durante la falla en A.

$Z_{\text {: }}$ Impedancia de la línea en Ohm por unidad de longitud

$d$ : Distancia de la falla (m)

Anteriormente, los métodos basados en la impedancia no consideraban la presencia de la GD [26]-[28]. Se han desarrollado algunas técnicas basadas en la impedancia para considerar la presencia de GD. Inicialmente estos métodos se basaban en la modificación o re-coordinación de los dispositivos de protección existentes en el SED y el objetivo era la identificación de la zona que presentaba la falla, pero sin una localización exacta de la misma [29]. Para determinar la localización exacta de la falla se han considerado diferentes parámetros, entre éstos variaciones en la tensión medida en la subestación y en las fuentes de GD antes y durante la falla [30], [31], uso de las componentes simétricas [32]-[35], entre otros. En la Tabla 5 se muestran los resultados de aplicación de los 
métodos basados en la impedancia para la localización de fallas en SED con GD.

Sin embargo, cuando un SED tiene diversos laterales con presencia de fuentes de GD, la exactitud de este método es cuestionable [36]. El hecho que un alimentador tenga diversos laterales aumenta la complejidad de localizar la falla. Esto se debe principalmente a que la estimación de la localización de la falla produce más de una ubicación [37]. De igual manera, el modelo de línea utilizado en el SED afecta la exactitud de estos métodos, en el caso de líneas de distribución cortas, las capacidades de derivación son despreciables y el modelo de línea corta es suficiente. Sin embargo, para líneas aéreas o subterráneas largas, el efecto de la admitancia de derivación es mayor y puede resultar en errores de estimación si no se modela correctamente. En general, un correcto modelado de las líneas del SED mejorará la exactitud de los resultados. Por otro lado, modelos detallados aumentarían la complejidad y la carga computacional [25]. En la Tabla 5 se muestran los resultados de aplicación de los métodos basados en la impedancia para la localización de fallas en SED con GD.

\subsection{Métodos basados en ondas viajeras}

Este método se basa en el principio de transmisión y reflexión de las ondas que se producen entre el terminal de línea y el punto de falla. En la Figura 3 se ejemplifica el método de las ondas viajeras. Cuando se produce una falla, se generan ondas de alta frecuencia de corrientes y tensiones que se propagan hacia ambos extremos. En los puntos de discontinuidad, como circuito abierto, cortocircuito o terminales de línea, una parte de la onda incidente se refleja hacia atrás mientras otra parte viaja hacia más allá de la discontinuidad. Las ondas se reflejan hacia adelante y atrás entre el punto de falla y los dos (2) terminales hasta que se alcanza el estado estacionario post-falla [25]. 


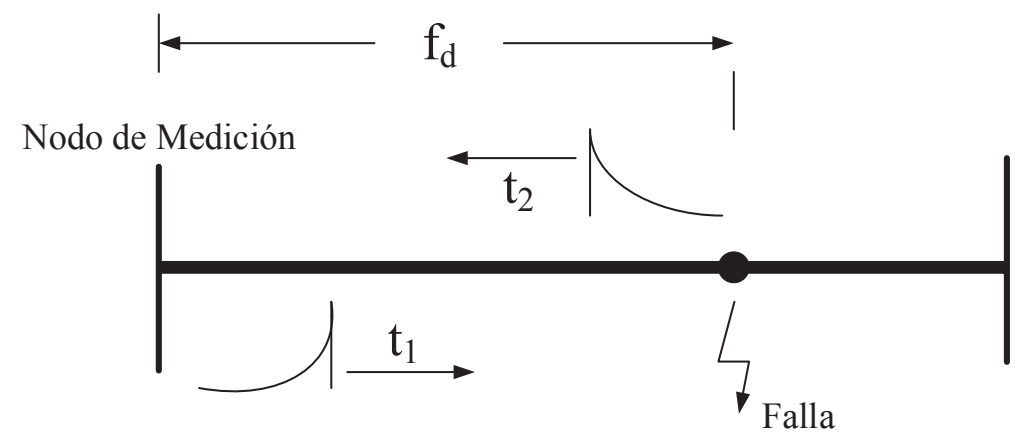

Figura 3. Método de las ondas viajeras

Fuente: [21]

En la Figura 3, $t_{1}$ es el tiempo que tarda la onda viajera en llegar al punto de falla desde el nodo de medición, $t_{2}$ es el tiempo que tarda la onda reflejada desde el punto de falla al nodo de medición y $f_{d}$ es la distancia de falla medida desde el punto de medición. La distancia de falla se determina usando (2)

$$
f_{d}=\frac{v *\left(t_{2}-t_{1}\right)}{2}
$$

Para cada localización de falla, los transitorios registrados contienen algunas frecuencias características dominantes en función de la longitud de las trayectorias de desplazamiento y de las velocidades de propagación que proporcionan información valiosa sobre su ubicación. Sin embargo, la interpretación de los transitorios, especialmente para SED ramificados, son computacionalmente difíciles [25]. Por lo tanto, los métodos basados en ondas viajeras se soportan principalmente en técnicas de procesamiento de señal tales como transformaciones de onda para simplificar el análisis, entre éstas se encuentra la transformada de Wavelet, la cual es una transformación lineal similar a la transformada de Fourier, que permite una representación tiempo-frecuencia de la señal. Esta propiedad es particularmente útil para la localización temporal de transitorios de falla que tienen componentes de alta frecuencia y de corta duración superpuestos a formas de onda continuas de frecuencia de potencia. 
El método de ondas viajeras requiere de dispositivos de adquisición de datos de alta velocidad, sensores, detectores de transitorios de falla y GPS para capturar la forma de onda transitoria para localizar la falla. La ventaja de este método es que no se ve afectada por la variación de la carga ni por altos valores de resistencia de conexión a tierra [21].

El método de ondas viajeras ha sido aplicado para la localización de fallas en SED con GD, basado en la transformada de Wavelet [38], [39] y en mediciones múltiples en el sistema [40]. En la Tabla 6 se muestran los resultados de aplicación de los métodos basados en ondas viajeras para la localización de fallas en SED con GD.

\section{3) Métodos basados en Inteligencia Artificial}

Debido a la complejidad de los SED y los diversos factores de incertidumbre, como la característica de los conductores (longitud y calibre) y el valor de la resistencia de falla, los cuales son difíciles de abordar con los métodos basados en la impedancia y las ondas viajeras, han aparecido métodos basados en el conocimiento para la localización de fallas en estos sistemas. En general, estos métodos requieren de patrones que caractericen la falla tales como las mediciones de tensión y corriente.

La Inteligencia Artificial (IA) es un subcampo de la informática que investiga cómo el pensamiento y la acción de los seres humanos pueden ser imitadas por las máquinas [41]. Tanto los cálculos numéricos, no numéricos y simbólicos están incluidas en el ámbito de la IA. La imitación de inteligencia incluye no sólo la capacidad de tomar decisiones racionales, sino también para hacer frente a información con datos faltantes, adaptarse a las situaciones existentes y mejorarse a sí misma en el horizonte a largo plazo sobre la base de la experiencia acumulada. Estos métodos basados en IA para localizar fallas en SED se mencionan a continuación.

\subsection{Redes neuronales}

Una Red Neuronal Artificial (ANN, por sus siglas en inglés) es un conjunto de neuronas primarias que están conectados juntas en 
diferentes arquitecturas organizadas en capas que están inspirada en la estructura del sistema nervioso de los seres humanos [41]. Las ANN son diferentes de los sistemas expertos, ya que no necesitan una base de conocimiento para trabajar. En su lugar, tienen que ser entrenadas con numerosos casos reales. Las ANN es uno de los métodos conocidos y confiables en la solución de problemas de ingeniería relacionados con la clasificación y optimización y por la capacidad que tienen en el reconocimiento de patrones complejos, su uso se ha extendido a la localización de fallas en SED. En la Figura 4 se presenta la estructura de una ANN feedfoward conocida como perceptron.

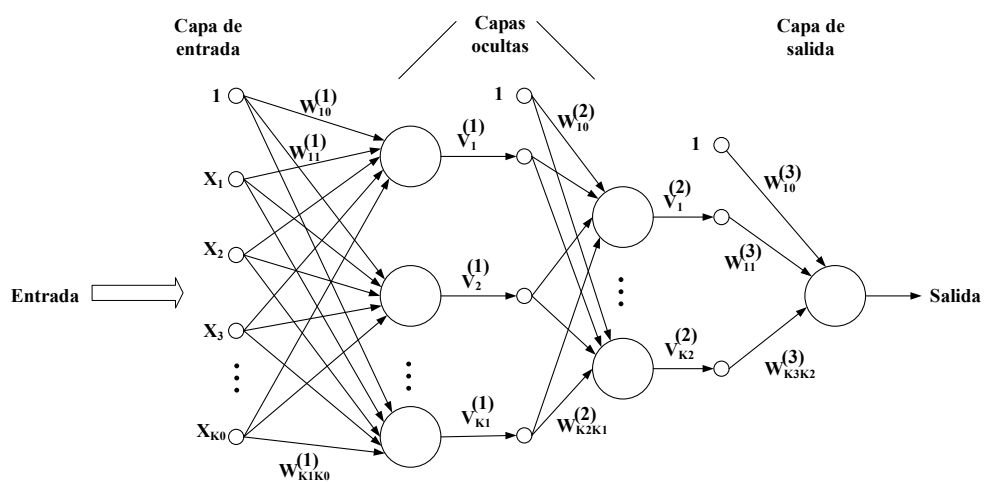

Figura 4. Representación gráfica de las ANN

Fuente: [41]

De la Figura 4 se distingue una capa de entrada la cual está compuesta por neuronas que reciben datos o señales procedentes del entorno. La capa de salida que está compuesta de neuronas que proporcionan la respuesta de la red neuronal. Una capa oculta que no tiene conexión directa con el entorno y proporciona grados de libertad a la red neuronal lo que le permite representar de manera más aproximada determinadas características del entorno que trata de modelar. Los datos primero ingresan a la capa de entrada luego pasan por la capa oculta y finalmente el resultado se obtiene en la capa de salida. Para la localización de fallas en sistemas eléctricos de distribución, los datos de entrada de la ANN pueden ser mediciones 
realizadas en el circuito, tales como tensión, corriente, el estado de los dispositivos de protección, entre otros. La salida de la ANN corresponde a la ubicación de la falla. En la Tabla 7 se presentan los resultados de la aplicación de las ANN en la localización de fallas en SED con GD.

La localización de fallas basada en ANN sufre de inexactitudes debido a las variaciones continuas de carga e impedancia de la fuente [41]. De igual manera, es altamente dependiente de la cantidad y calidad de los datos entrenados para producir un adecuado algoritmo, por lo tanto, una cantidad limitada de información afecta la eficiencia del método. Este problema ocurre en los SED con información limitada resultante de un número insuficiente de dispositivos de monitoreo. Otra desventaja de las ANN es que el proceso de entrenamiento tiene convergencia lenta. Además, los parámetros como las capas ocultas, las neuronas y la tasa de aprendizaje se identifican utilizando casos de prueba y error. De igual manera, las ANN deben ser reentrenadas cada vez que el SED experimente cambios [21].

\subsection{Máquinas de soporte vectorial}

Las bases de esta herramienta fueron desarrolladas por Vladimir Vapnik [42] y son populares debido a muchas características atractivas y prometedoras que presenta. La formulación incorpora la Minimización del Riesgo Estructural (SRM, por sus siglas en inglés) que se ha demostrado ser superior al principio de Minimización del Riesgo Empírico (ERM, por sus siglas en inglés), empleado por las ANN convencionales [43]. La SRM minimiza un límite superior en el riesgo esperado, en contraposición a ERM que minimiza el error en los datos de entrenamiento. Es esta diferencia la que equipa las SVM con una mayor capacidad de generalizar, que es el objetivo en el aprendizaje estadístico. Las SVM se han desarrollado para resolver el problema de clasificación y regresión [44].

El concepto de las SVM se muestra en la Figura 5 para la clasificación entre dos (2) clases (clase 1 y clase 2 ). Los puntos se marcan usando el conjunto de entrenamiento de los datos de tensión y/o corriente tomados en el nodo de medición para la clasificación de fallas. Los vectores de soporte son los elementos de los conjuntos 
de entrenamiento, que identifican el Hiperplano de Separación Óptima (OSH, por sus siglas en inglés). Los círculos representan la clase 1 y los cuadrados la clase 2 . La entrada para las clases 1 y 2 son del nodo de medición (tensión y/o corriente) y la salida es la ubicación de la falla. El OSH separa todas las muestras y también maximiza el margen entre los dos (2) planos paralelos que contienen el conjunto de clases. El margen se define como la suma de la distancia mínima entre el conjunto de datos de entrenamiento y el OSH. En la figura 5 se muestra la representación gráfica de las SVM. De (3) a (6) se muestran las ecuaciones de los planos paralelos al OSH, el margen y la distancia al origen.

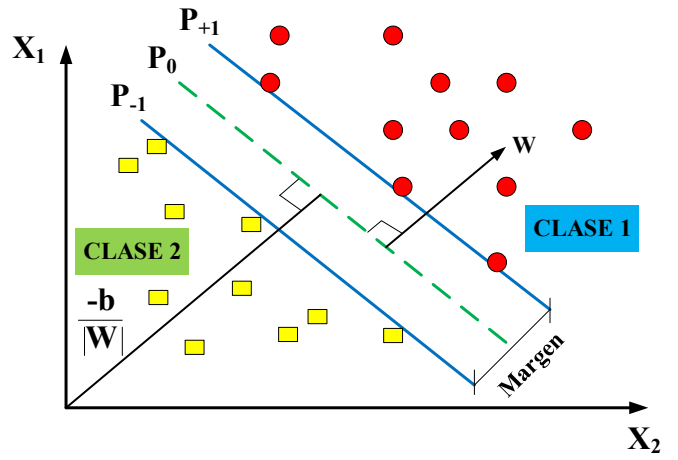

Figura 5. Representación gráfica de las SVM

$$
\begin{gathered}
P_{+1}:\left(\vec{w} \cdot \overrightarrow{x_{l}}\right)+b=+1 \\
P_{-1}:\left(\vec{w} \cdot \overrightarrow{x_{l}}\right)+b=-1 \\
\frac{\vec{w}}{\|\vec{w}\|} \cdot\left(\overrightarrow{x_{+1}}-\overrightarrow{x_{-1}}\right)=\frac{2}{\|\vec{w}\|} \\
\frac{\left(\vec{w} \cdot \overrightarrow{x_{l}}\right)+b}{\|\vec{w}\|}=\frac{b}{\|\vec{w}\|}
\end{gathered}
$$

Para encontrar el OSH se debe maximizar el margen (5), considerando la restricción de que no existan datos dentro del margen, lo q

$$
\min _{w} \frac{1}{2}(\vec{w} \cdot \vec{w})
$$




$$
\text { sujeto a } y_{i}\left(\vec{w} \cdot \overrightarrow{x_{l}}+b\right) \geq 1, \forall i
$$

La ecuación (7) se llama función objetivo y, junto con (8), representa el problema de optimización cuadrático con restricciones, lo que puede ser resuelto utilizando el método de los multiplicadores de Lagrange, denotados por la ecuación (9)

$$
L(\vec{w}, b, \vec{\alpha})=\frac{1}{2}\|\vec{w}\|^{2}-\sum_{i=1}^{n} \alpha_{i}\left[y_{i}\left(\vec{w} \cdot \overrightarrow{x_{\imath}}+b\right)-1\right]
$$

Para el caso de que existan intersecciones en los datos de entrenamiento (datos no separables), tal como se muestra en la Figura 6, los puntos que se encuentran en la intersección no pueden ser clasificados correctamente y sus correspondientes tenderán al infinito.

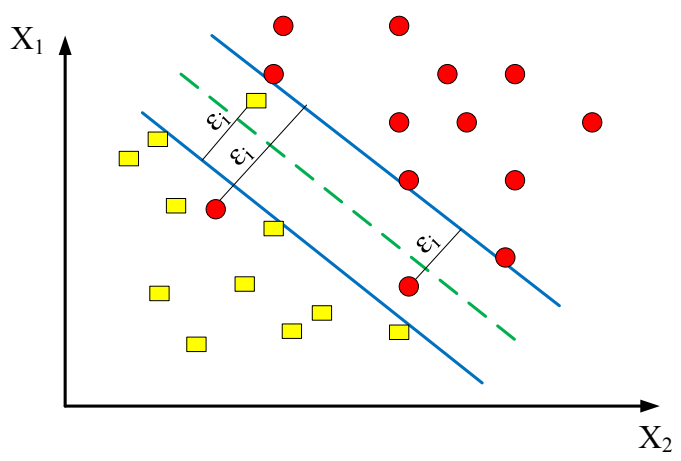

Figura 6. Hiperplano clasificador óptimo para caso no separable

En [42] introducen las variables slack o de relajación para permitir datos que violen las restricciones. De esta manera para obtener el OSH corresponde a minimizar la expresión (10)

$$
\min _{w}\left[\frac{1}{2}(\vec{w} \cdot \vec{w})+C \sum_{i=1}^{n} \xi_{i}\right]
$$

Sujeto a $y_{i}\left(\vec{w} \cdot \overrightarrow{x_{l}}+b\right) \geq 1-\xi_{i}, \forall i$ 
El parámetro $\mathrm{C}$ se elige por conveniencia por el usuario, de tal manera que, un valor grande sea una alta penalización a los errores. El valor óptimo de $\mathrm{C}$ se obtiene por medio de una validación cruzada [45].

Cuando los datos no son separables, se mapea el espacio de entrada a un espacio de representación de una dimensión mayor a través de una función no lineal elegida por conveniencia [46], tal como se muestra en la figura 7.

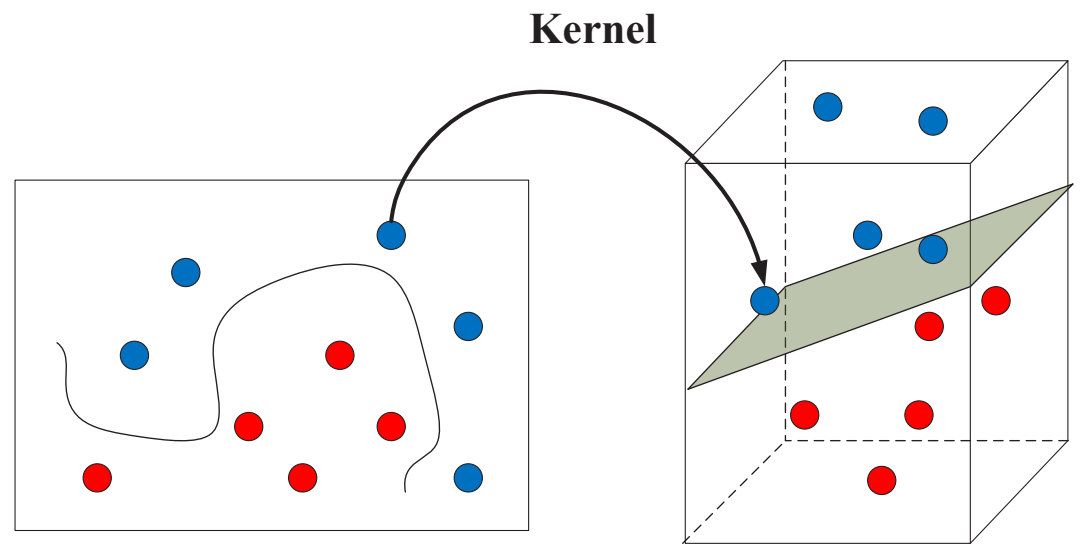

Figura 7. Transformación del espacio de entrada al espacio característico

Por medio de una función $(\Phi)$ se trazan los datos de entrada a algún espacio de mayor dimensión donde se puede manejar una separación lineal. Este espacio es llamado espacio característico (F) y es denotado por la relación (11).

$$
\Phi: R^{N} \rightarrow F
$$

La función $(\Phi)$ se define como el producto punto de los vectores en el espacio característico tal como se muestra en la ecuación (12).

$$
k\left(\vec{x}_{i}, \overrightarrow{x_{\jmath}}\right)=\Phi\left(\vec{x}_{l}\right) \cdot \Phi\left(\vec{x}_{\jmath}\right)
$$

La ecuación (12) se conoce también como la función Kernel (k) y entre las más usadas se encuentran la lineal, la RBF, la polinomial y la sigmoide. Estas funciones se muestran en la Tabla 8. 
Tabla 8. Funciones Kernel más usadas

\begin{tabular}{|c|c|}
\hline Kernel & Función \\
\hline Lineal & $K\left(X_{i}, X_{j}\right)=X_{i}^{T} X_{j}$ \\
\hline RBF & $K\left(X_{i}, X_{j}\right)=\exp \left(-\gamma\left\|X_{i}-X_{j}\right\|^{2}\right), \gamma>0$ \\
\hline Polinomial & $K\left(X_{i}, X_{j}\right)=\left(\gamma X_{i}^{T} X_{j}+r\right)^{d}, \gamma>0$ \\
\hline Sigmoide & $K\left(X_{i}, X_{j}\right)=\tanh \left(\gamma X_{i}^{T} X_{j}+r\right)$ \\
\hline
\end{tabular}

$\gamma, r$ y d son los parámetros de la función kernel.

Fuente: [45]

Una SVM es un algoritmo de aprendizaje utilizado típicamente para resolver problemas de clasificación binaria y luego se extendieron para tratar problemas con múltiples clases. Estas múltiples clases son las que se presentan en la localización de fallas en SED y se resuelve por medio del esquema propuesto por [47]. En la Tabla 9 se presentan los resultados de la aplicación de las SVM en la localización de fallas en SED con GD.

\section{Métodos integrados}

Se dan tres (3) tipos de métodos para localizar las fallas en SED conocidos como la inspección visual, el basado en la impedancia y las ondas viajeras y los métodos inteligentes. Sin embargo, éstos todavía presentan algunos problemas, por ejemplo, el método basado en ondas viajeras puede enfrentarse a problemas tales como alta frecuencia de muestreo y estructura compleja, los métodos inteligentes pueden ser problemáticos debido a la necesidad de un banco de datos grande y detallado [48]. Finalmente, los métodos basados en la impedancia presentan problemas de múltiple estimación como se menciona en apartados anteriores.

El siguiente método consiste en la combinación de más de un método de localización de fallas y permite aprovechar la fortaleza de cada uno de éstos y así se podría obtener una mejor exactitud, este se conoce como método integrado [10]. En la Tabla 10 se muestra los métodos integrados para la localización de fallas en SED. Cabe destacar que tales combinaciones, además de sus ventajas, también traen desventajas. Si bien, la ventaja común de los mencionados 
métodos integrados es su capacidad para superar el problema de estimación múltiple, tienen requerimientos adicionales en comparación con los algoritmos basados en impedancia tales como datos de entrenamiento, mediciones con mayor frecuencia de muestreo, medidas dispersas de tensión, por mencionar algunos. Para finalizar, en la Tabla XI se presenta una comparación de los diferentes métodos propuestos de localización de fallas en SED con GD.

\section{Conclusiones}

La localización de fallas en SED con GD es una problemática que ha sido abordada con diferentes técnicas, las cuales tienen sus ventajas y desventajas. Estas técnicas abarcan la inspección visual, los métodos convencionales, la inteligencia artificial y los métodos integrados. En esta investigación se presenta una revisión de los principales métodos utilizados para tratar el problema. Cada uno de ellos presenta sus ventajas y desventajas con errores de estimación aceptables. Se evidencia una tendencia a integrar los métodos existentes para mejorar los resultados. La presencia de GD en los SED extiende las investigaciones del tema debido a los efectos directos que tiene sobre las metodologías convencionales que daban solución al problema de localización de fallas en SED. 


\begin{tabular}{|c|c|c|c|}
\hline \multirow{8}{*}{ 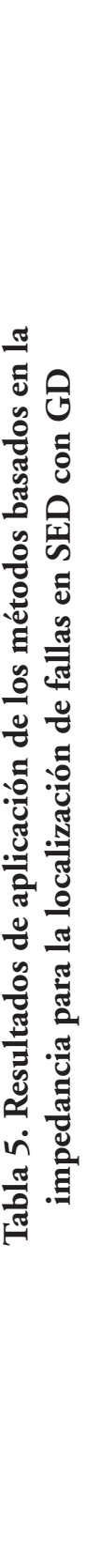 } & 䒕 & 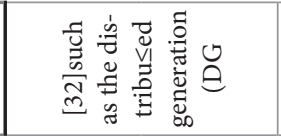 & 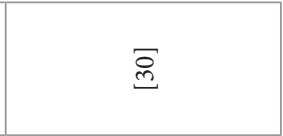 \\
\hline & 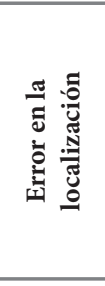 & 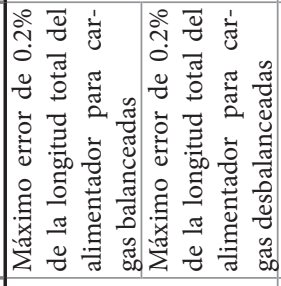 & 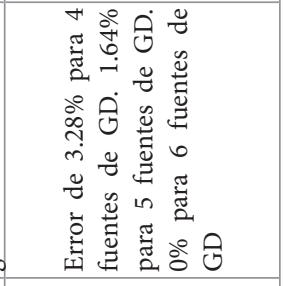 \\
\hline & 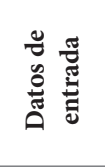 & 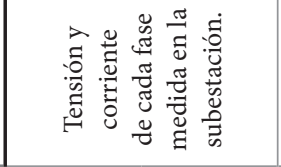 & 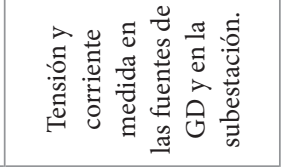 \\
\hline & 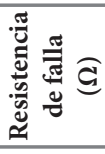 & 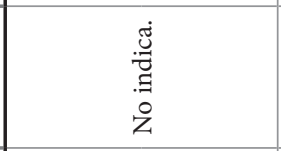 & 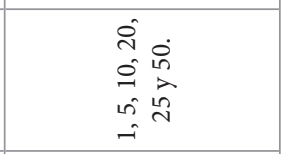 \\
\hline & 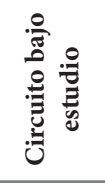 & 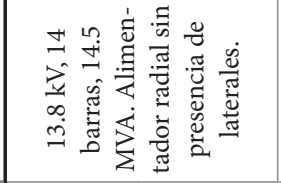 & 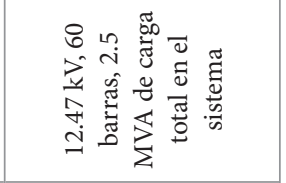 \\
\hline & 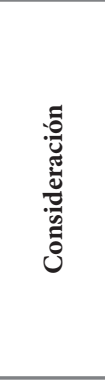 & 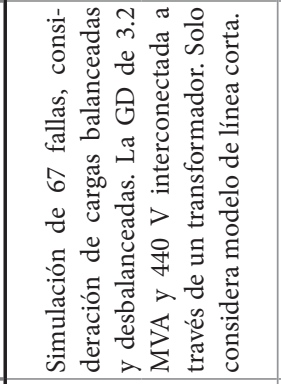 & 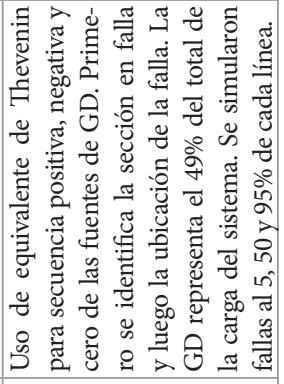 \\
\hline & 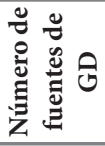 & - & 6 \\
\hline & 兽 & 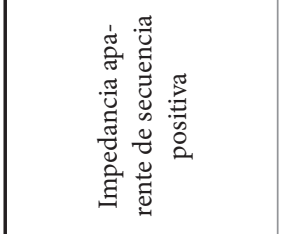 & 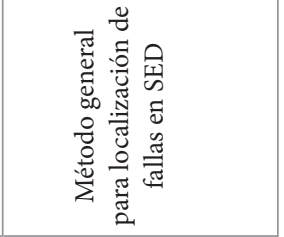 \\
\hline
\end{tabular}




\begin{tabular}{|c|c|c|}
\hline 苞 & $\stackrel{g}{+}$ & 号 \\
\hline 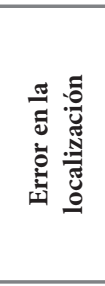 & 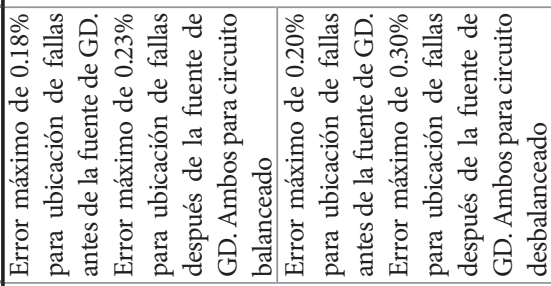 & 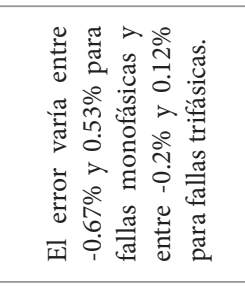 \\
\hline 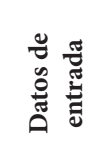 & 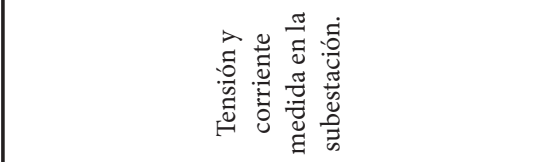 & 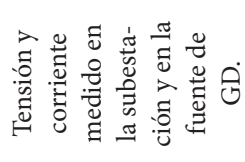 \\
\hline 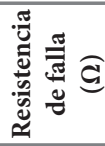 & $\begin{array}{l}\tilde{\sigma} \\
\stackrel{0}{0} \\
0 \\
0 \\
0\end{array}$ & 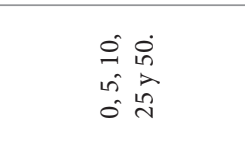 \\
\hline 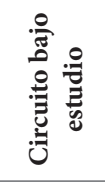 & 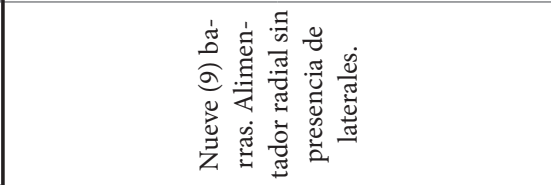 & 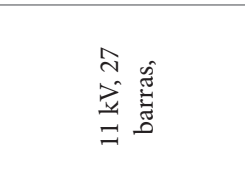 \\
\hline 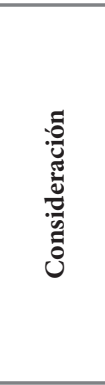 & 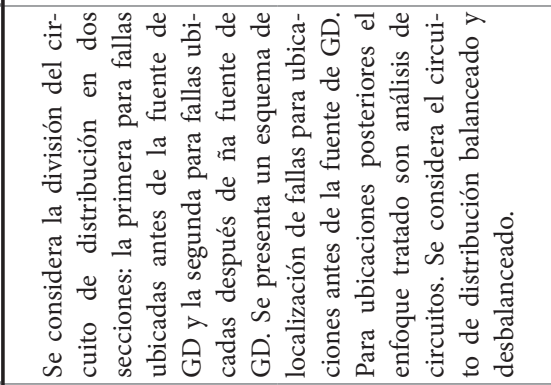 & 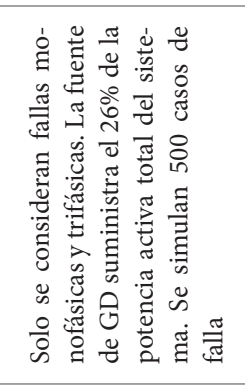 \\
\hline 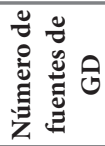 & 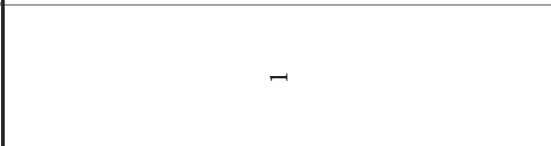 & $\neg$ \\
\hline 总 & 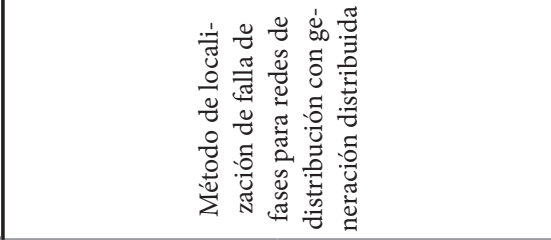 & 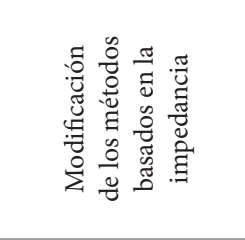 \\
\hline
\end{tabular}




\begin{tabular}{|c|c|}
\hline 泀 & 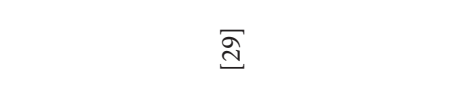 \\
\hline 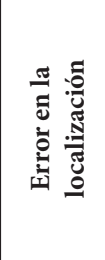 & 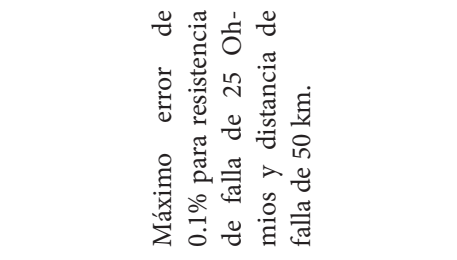 \\
\hline 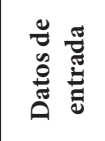 & 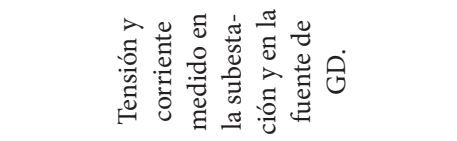 \\
\hline 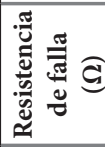 & $\begin{array}{l}\hat{0} \\
\overrightarrow{0} \\
0\end{array}$ \\
\hline 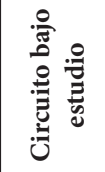 & 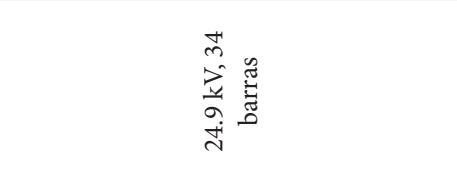 \\
\hline 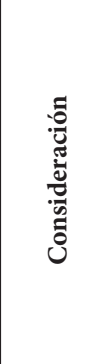 & 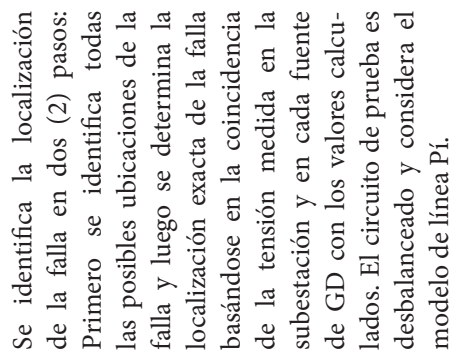 \\
\hline 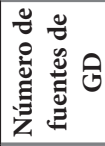 & $N$ \\
\hline 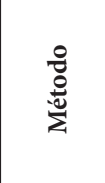 & 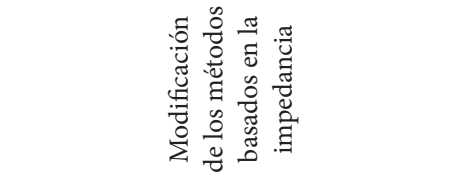 \\
\hline
\end{tabular}

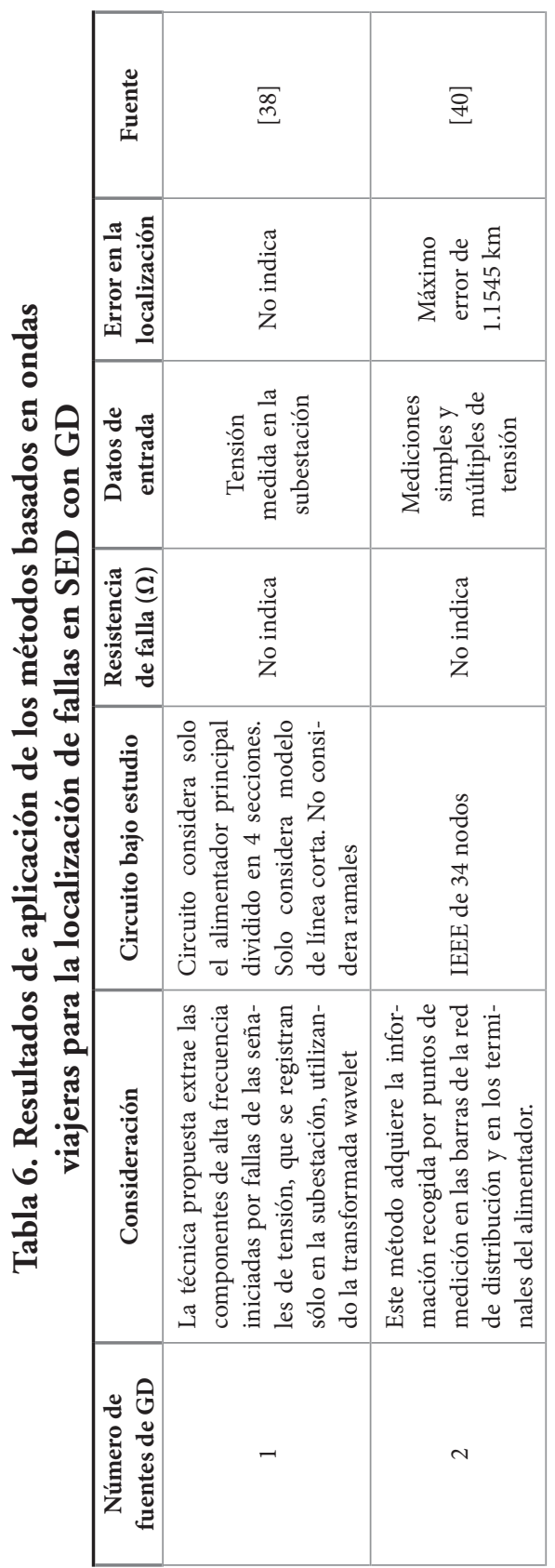




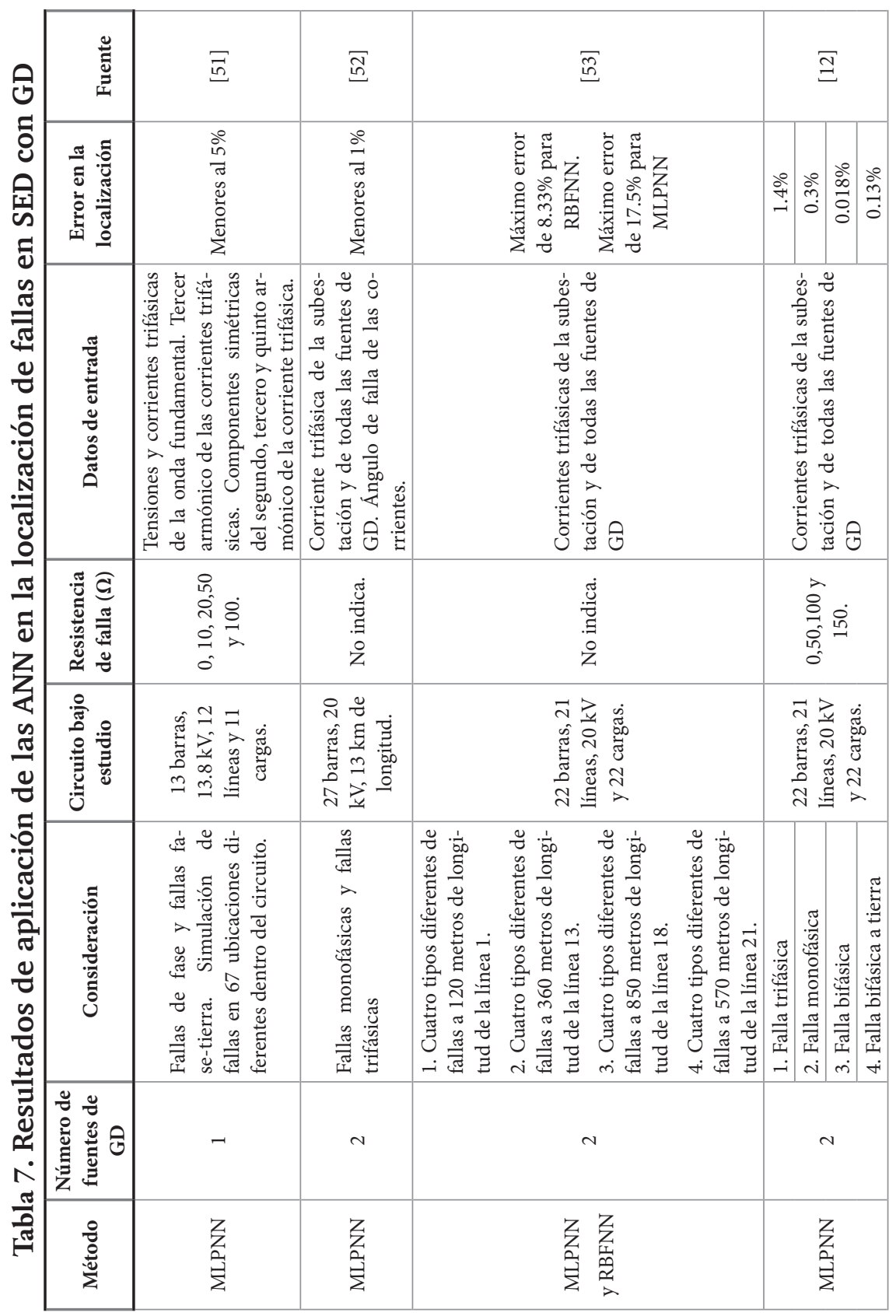




\begin{tabular}{|c|c|c|c|c|c|c|c|}
\hline 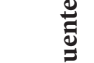 & \multicolumn{7}{|c|}{$\underset{n}{F}$} \\
\hline 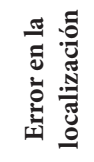 & लें & $\mid \begin{array}{c}0 \\
\dot{0} \\
\dot{+} \\
\dot{0} \\
i\end{array}$ & \begin{tabular}{l}
$\stackrel{0}{\dagger}$ \\
\multirow{j}{*}{} \\
$\dot{0}$ \\
$\dot{r}$
\end{tabular} & 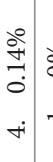 & 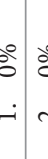 & 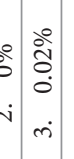 & $\begin{array}{l}\stackrel{\circ}{\circ} \\
\stackrel{+}{0} \\
\dot{0} \\
\dot{+}\end{array}$ \\
\hline 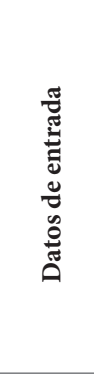 & \multicolumn{7}{|c|}{ 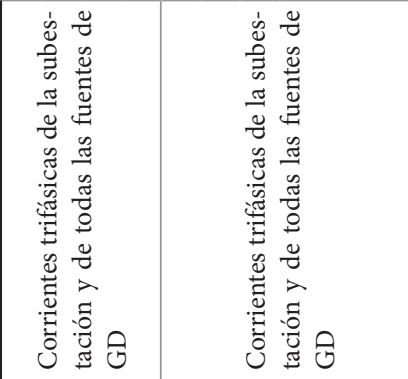 } \\
\hline 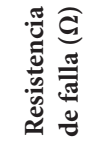 & \multicolumn{7}{|c|}{ 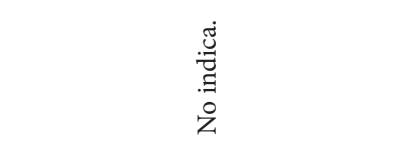 } \\
\hline 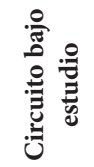 & \multicolumn{7}{|c|}{ 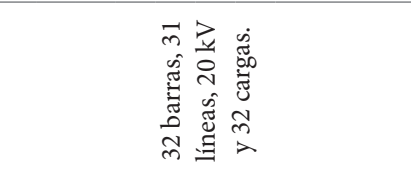 } \\
\hline 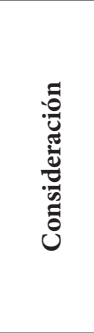 & & $\begin{array}{l}\frac{\pi}{0} \\
0 \\
0 \\
0 \\
0 \\
0 \\
0 \\
0 \\
0 \\
0 \\
0 \\
0 \\
0 \\
0 \\
0 \\
0 \\
0 \\
0 \\
0\end{array}$ & 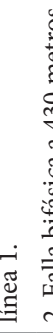 & 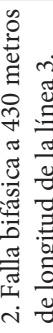 & 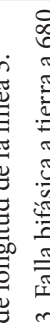 & 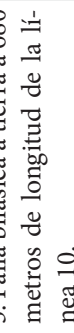 & 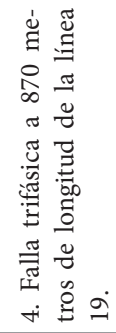 \\
\hline 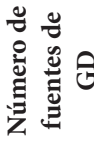 & & & & & 0 & & \\
\hline 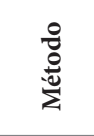 & & 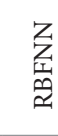 & & & & & \\
\hline
\end{tabular}




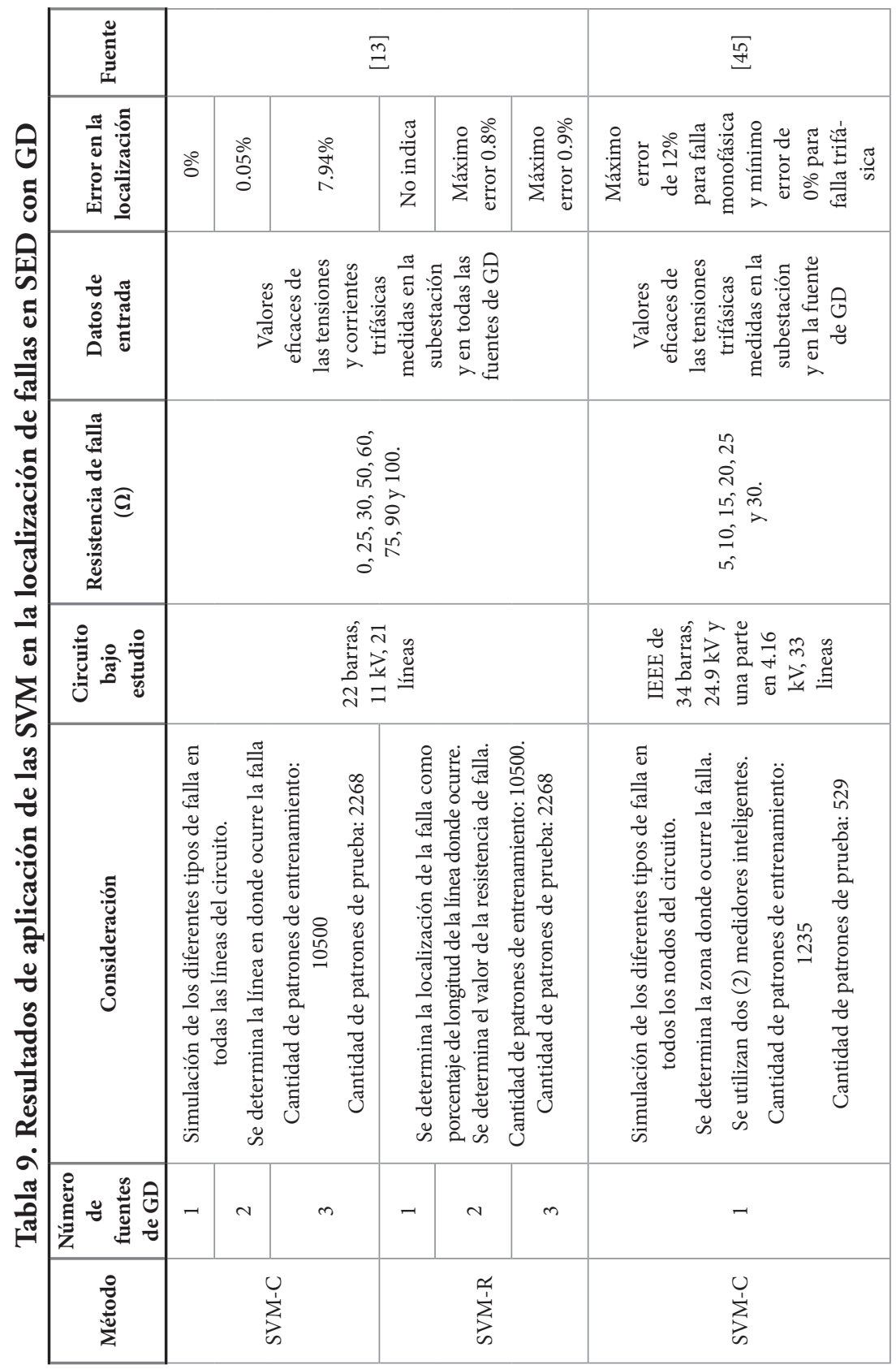




\begin{tabular}{|c|c|c|c|c|c|c|}
\hline 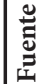 & 资 & 뉸 & in & 冓 & हn & 8 \\
\hline 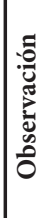 & 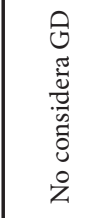 & 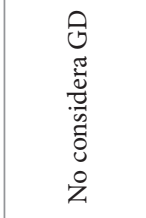 & 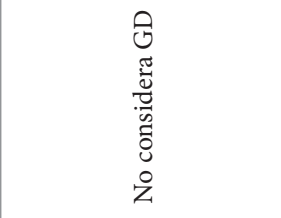 & 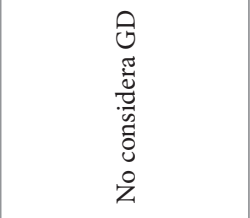 & 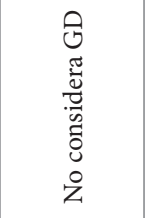 & 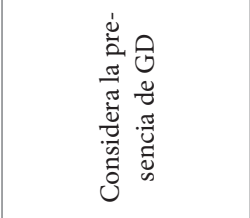 \\
\hline 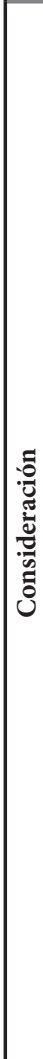 & 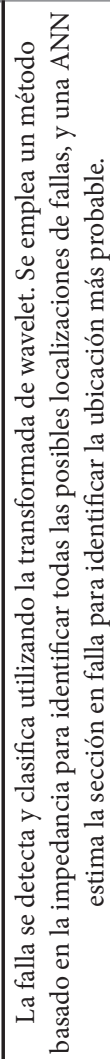 & 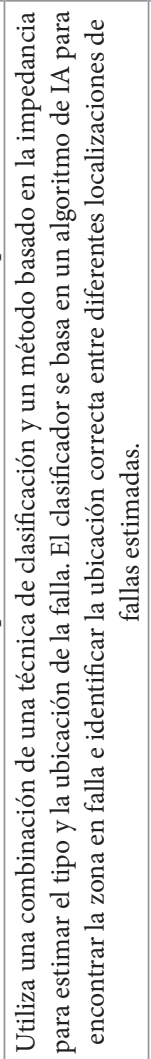 & 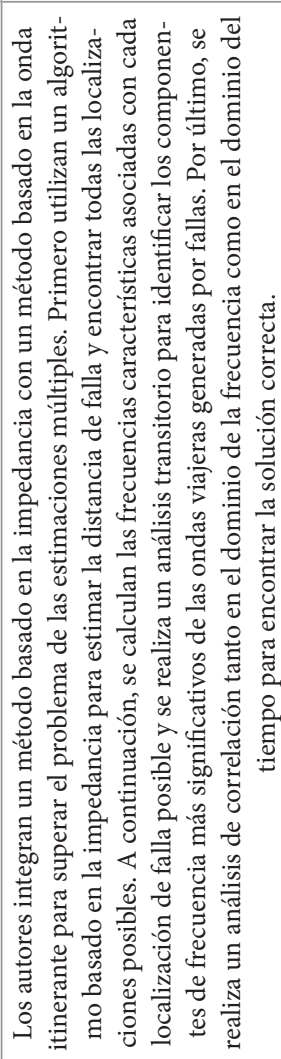 & 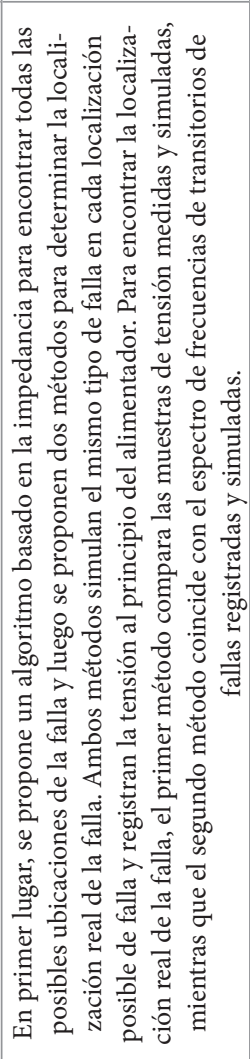 & 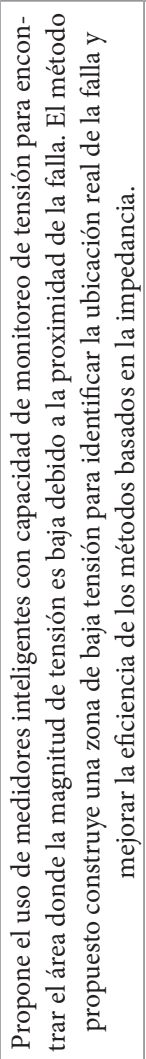 & 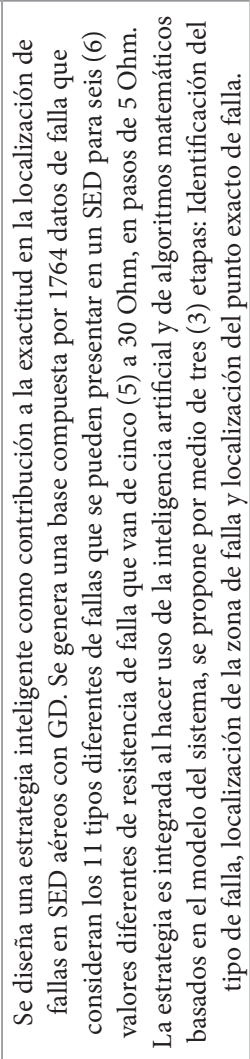 \\
\hline 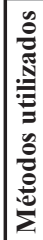 & 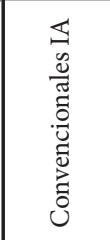 & 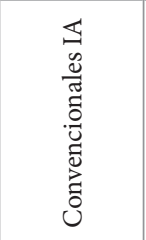 & 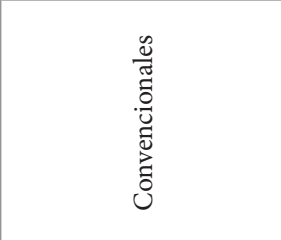 & 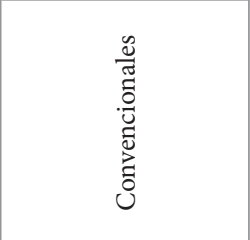 & 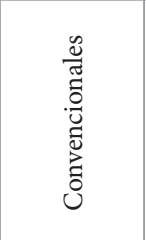 & 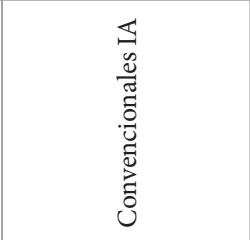 \\
\hline
\end{tabular}




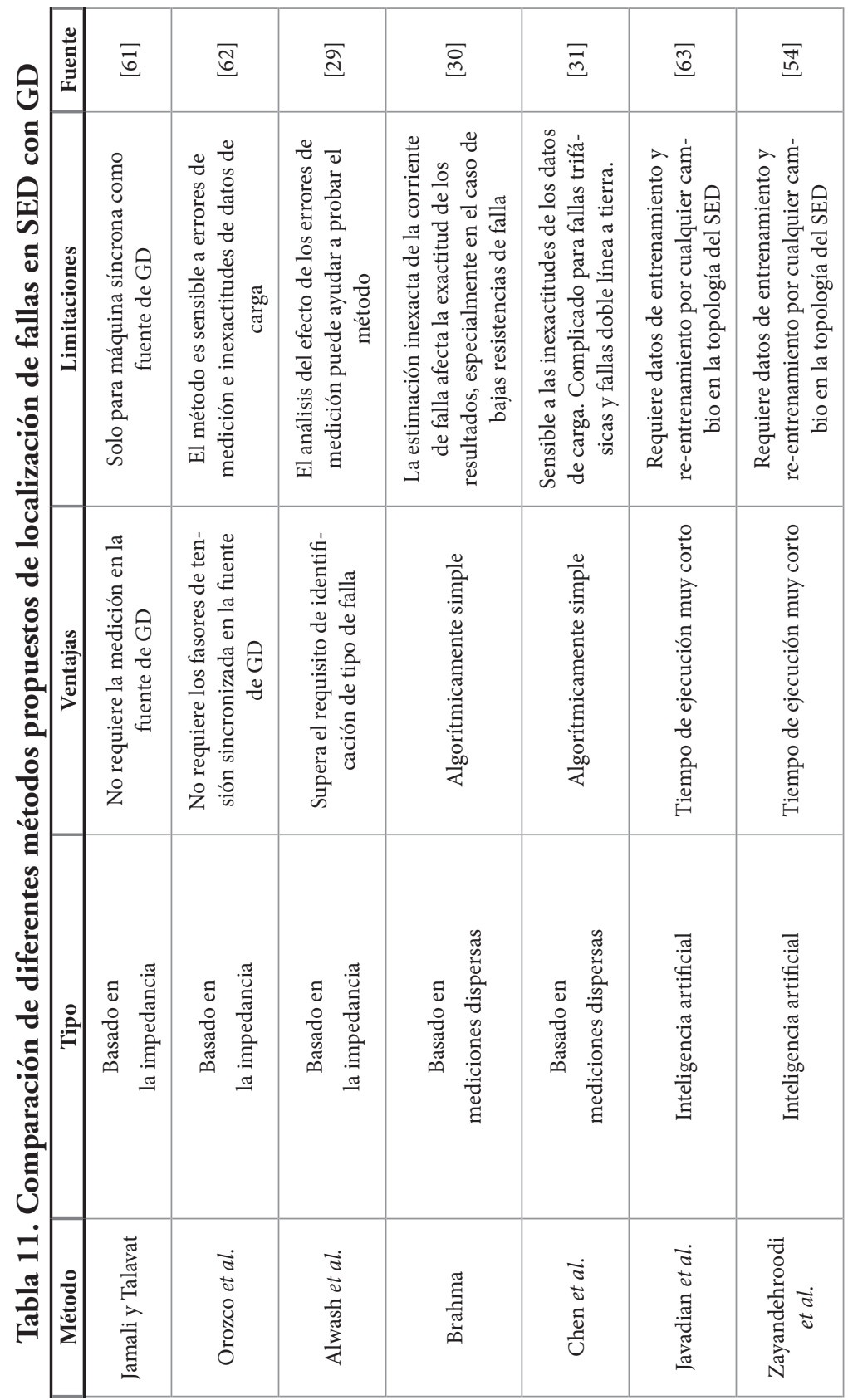




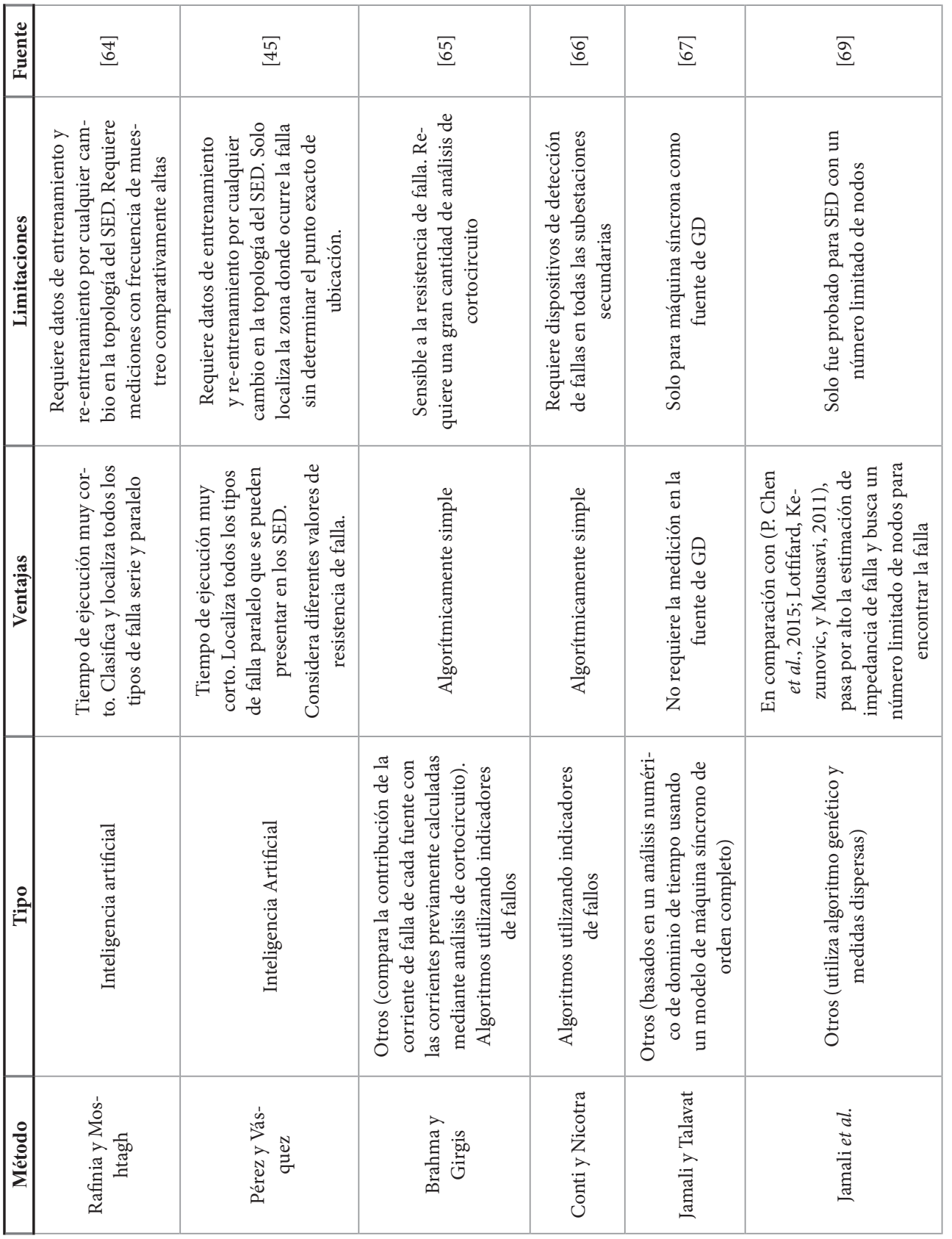




\section{Referencias}

[1] T. Short, Electric power distribution: handbook, vol. 10. 2004.

[2] S. Ramirez, Redes de Distribución de Energía, 3.a ed. Manizales, 2004.

[3] M. Kezunovic, «Smart Fault Location for Smart Grids», IEEE Transactions on Smart Grid, vol. 2, n.o 1, pp. 11-22, 2011.

[4] P. Mohammadi, E. Kishyky, A. Akher, y A. Salam, «The impacts of distributed generation on fault detection and voltage profile in power distribution networks», International Power Modulator and High Voltage Conference (IPMHVC), pp. 191-196, 2014.

[5] Y. Menchafou, H. Markhi, M. Zahri, y M. Habibi, «Impact of distributed generation integration in electric power distribution systems on fault location methods», 3rd International Renewable and Sustainable Energy Conference (IRSEC), n.o 1998, 2015.

[6] A. Fazanehrafat, S. Javadian, S. Bathaee, y M. Haghifam, «Maintaining the recloser-fuse coordination in distribution systems in presence of DG by determining DG's size», IET 9th International Conference on Developments in Power Systems Protection (DPSP 2008), pp. 132-137, 2008.

[7] B. José, P. Cavalcante, F. Trindade, y M. Almeida, «Analysis of Distance Based Fault Location Methods for Smart Grids with Distributed Generation», 2013 4th IEEE PES Innovative Smart Grid Technologies Europe (ISGT Europe), pp. 1-6, 2013.

[8] C. Orozco, J. Mora, y S. Pérez, «Método de localización de fallas basado en impedancia aparente para sistemas de distribución con generación distribuida», Ingeniare Revista chilena de ingeniería, vol. 23, n.o 3, pp. 348-360, 2015.

[9] J. Mora, J. Meléndez, y G. Carrillo, «Comparison of impedance based fault location methods for power distribution systems», Electric Power Systems Research, vol. 78, n.o 4, pp. 657-666, 2008.

[10] L. Awalin, H. Mokhlis, y A. Bakar, «Recent developments in fault location methods for distribution networks», Przeglad Elektrotechniczny, n.o 12, pp. 206-212, 2012.

[11] M. Mirzaei, M. Kadir, E. Moazami, y H. Hizam, «Review of Fault Location Methods for Distribution Power System», Australian Journal of Basic and Applied Sciences, vol. 3, n.o 3, pp. 2670-2676, 2009.

[12] S. Javadian y M. Massaeli, «A fault location method in distribution networks including DG», Indian Journal of Science and Technology, vol. 4, n.o 11, pp. 1446-1451, 2011.

[13] R. Agrawal y D. Thukaram, «Identification of fault location in power distribution system with distributed generation using support vector machines», 2013 IEEE PES Innovative Smart Grid Technologies Conference (ISGT), pp. 1-6, 2013.

[14] M. Torres, «Contribución de la información en el diseño de una aproximación de la evaluación de la calidad del servicio», Trabajo de Tesis Doctoral 
presentada para optar al grado de Doctor en Ciencias de la Ingeniería mención Productividad, Universidad Experimental Politécnica" Antonio José de Sucre”, UNEXPO Barquisimeto, 2010.

[15] R. Das, «Determining the locations of faults», University of Saskatchewan, 1998.

[16] C. Vásquez, «Criterios de eficiencia para disminuir las pérdidas debidas a la no calidad de la energía eléctrica», en Trabajo de Ascenso presentado ante la Universidad Nacional Experimental Politécnica «Antonio José de Sucre» como requisito para ascender a la Categoría de Profesora Titular, Barquisimeto, 2011, pp. 1-164.

[17] IEEE Power \& Energy Society, «IEEE Recommended Practice for Monitoring Electric Power Quality», IEEE Std 1159, 2009.

[18] J. Burke y D. Laurence, «Characteristics of fault currents on distribution systems», IEEE Transactions on Power Apparatus and Systems, n.o 1, pp. 1-6, 1984.

[19] H. Farooq, C. Zhou, M. E. Farrag, y M. Ejaz, «Investigating the impacts of distributed generation on an electrical distribution system already stressed by non-linear domestic loads», Asia-Pacific Power and Energy Engineering Conference, APPEEC, vol. 100, n.o 1, pp. 2-5, 2012.

[20] T. Ackermann, G. Andersson, y L. Söder, «Distributed generation: A definition», Electric Power Systems Research, vol. 57, n.o 3, pp. 195-204, 2001.

[21] S. Gururajapathy, H. Mokhlis, y H. Illias, «Fault location and detection techniques in power distribution systems with distributed generation: A review», Renewable and Sustainable Energy Reviews, vol. 74, n.o February 2016, pp. 949-958, 2017.

[22] R. Acevedo, «Análisis de la evolución del marco legal del servicio eléctrico venezolano en el período 2000 al 2010», Gestión y Gerencia, vol. 5, n.o 3, pp. 104-122, 2010.

[23] J. Montenegro y A. Urdaneta, «Evolución histórica del marco regulatorio del sector eléctrico latinoamericano», Interciencia, vol. 39, n.o 3, pp. 164-171, 2014.

[24] IEEE Power Engineering Society, «IEEE Guide for Electric Power Distribution Reliability Indices», IEEE Std 1366, pp. 1-21, 1998.

[25] A. Bahmanyar, S. Jamali, A. Estebsari, y E. Bompard, «A comparison framework for distribution system outage and fault location methods», Electric Power Systems Research, vol. 145, pp. 19-34, 2017.

[26] D. Novosel, D. Hart, Y. Hu, y J. Myllymaki, «System for locating faults and estimating fault resistance in distribution networks with tapped loads», 1998.

[27] M. Saha y E. Rosolowski, «Method and device of fault location for distribution networks, US Patent number 6,483,435», 2002.

[28] J. Zhu, D. Lubkeman, y A. Girgis, «Automated fault location and diagnosis on electric power distribution feeders», Power Delivery, IEEE Transactions, vol. 12, n.o 2, pp. 801-809, 1997. 
[29] S. Alwash, V. Ramachandaramurthy, y N. Mithulananthan, «Fault-Location Scheme for Power Distribution System with Distributed Generation», IEEE Transactions on Power Delivery, vol. 30, pp. 1187-1195, 2015.

[30] S. Brahma, «Fault location in power distribution system with penetration of distributed generation», IEEE Transactions on Power Delivery, vol. 26, n.o 3, pp. 1545-1553, 2011.

[31] P. Chen, V. Malbasa, Y. Dong, y M. Kezunovic, «Sensitivity Analysis of Voltage Sag Based Fault Location With Distributed Generation», IEEE Transactions on Smart Grid, vol. 6, n.o 4, pp. 2098-2106, 2015.

[32] A. Bretas y R. Salim, «Fault location in unbalanced dg systems using the positive sequence apparent impedance», 2006 IEEE PES Transmission and Distribution Conference and Exposition: Latin America, TDC'06, vol. 00, pp. 1-6, 2006.

[33] J. Nunes y A. Bretas, «A impedance-based fault location technique for unbalanced distributed generation systems», 2011 IEEE PES Trondheim PowerTech: The Power of Technology for a Sustainable Society, POWERTECH 2011, pp. 1-7, 2011.

[34] J. Nunes y A. Bretas, «Impedance-based fault location formulation for unbalanced primary distribution systems with distributed generation», 2010 International Conference on Power System Technology: Technological Innovations Making Power Grid Smarter, POWERCON2010, 2010.

[35] D. Penkov, B. Raison, C. Andrieu, J. P. Rognon, y B. Enacheanu., «DG impact on three fault location. DG use for fault location purposes?», International Conference on Future Power Systems, vol. 4, n.o December, p. 6, 2005.

[36] J. Cordova y O. Faruque, «Fault location identification in smart distribution networks with distributed generation», 2015.

[37] F. Dehghani y H. Nezami, «A new fault location technique on radial distribution systems using artificial neural network», 22nd International Conference on Electricity Distribution, pp. 10-13, 2013.

[38] A. Abur, «Fault Location in Distribution Systems with Distributed Generation», en 15th PSCC, 2005, pp. 1-5.

[39] X. Chen, X. Yin, y S. Deng, «A novel method for SLG fault location in power distribution system using time lag of travelling wave components», IEEJ Transactions on Electrical and Electronic Engineering, vol. 12, n.o 1, pp. 45-54, 2017.

[40] X. Chen, X. Yin, X. Yin, J. Tang, y M. Wen, «A novel traveling wave based fault location scheme for power distribution grids with distributed generations», 2015 IEEE Power \& Energy Society General Meeting, pp. 1-5, 2015.

[41] M. Saha, J. Izykowski, y E. Rosolowski, Fault Location on Power Networks, 1.a ed. Londrés, 2010.

[42] C. Cortes y V. Vapnik, «Support-Vector Networks», Machine Learning, vol. 20, n.o 3, pp. 273-297, 1995. 
[43] S. R. Gunn, «Support Vector Machines for Classification and Regression», Image Speech and Intelligent Systems Technical Report, vol. 14, n.o May, pp. 230-67, 1998.

[44] V. Vapnik, S. E. Golowich, y A. Smola, «Support Vector Method for Function Approximation, Regression Estimation, and Signal Processing», Annual Conference on Neural Information Processing Systems (NIPS), pp. 281-287, 1996.

[45] R. Pérez y C. Vásquez, «Fault Location in Distribution Systems with Distributed Generation Using Support Vector Machines and Smart Meters», IEEE Ecuador Technical Chapters Meeting (ETCM), pp. 1-6, 2016.

[46] B. E. Boser, I. M. Guyon, y V. N. Vapnik, «A Training Algorithm for Optimal Margin Classifiers», Proceedings of the Fifth Annual ACM Workshop on Computational Learning Theory, pp. 144-152, 1992.

[47] C. Hsu y C. Lin, «A comparison of methods for multiclass support vector machines», IEEE Transactions on Neural Networks, vol. 13, n.o 2, pp. 415425, 2002.

[48] M. Daisy y R. Dashti, «Single phase fault location in electrical distribution feeder using hybrid method», Energy, vol. 103, pp. 356-368, 2016.

[49] M. Hagh, M. Hosseini, y S. Asgarifar, «A novel phase to phase fault location algorithm for distribution network with distributed generation», CIRED Workshop, pp. 1-4, 2012.

[50] F. Abo, M. Alaam, y A. Azmy, «Impedance-based fault location technique for distribution systems in presence of distributed generation», IEEE International Conference on Smart Energy Grid Engineering, SEGE 2013, pp. 28-30, 2013.

[51] A. Bretas, M. Moreto, R. Salim, y L. Pires, «A Novel High Impedance Fault Location for Distribution Systems Considering Distributed Generation», 2006 IEEE/PES Transmission \& Distribution Conference and Exposition: Latin America, n.o September, pp. 1-6, 2006.

[52] G. Shahgholian y M. Rezaei, «Fault Location Scheme in Distribution Systems with Distributed Generators Using Neural Networks», Majlesi Journal of Electrical Engineering, vol. 4, n.o 2, pp. 57-62, 2010.

[53] H. Zayandehroodi, A. Mohamed, H. Shareef, y M. Mohammadjafari, «Performance comparison of mlp and rbf neural networks for fault location in distribution networks with DGs», 2010 IEEE International Conference on Power and Energy, pp. 341-345, 2010.

[54] H. Zayandehroodi, A. Mohamed, M. Farhoodnea, y M. Mohammadjafari, «An optimal radial basis function neural network for fault location in a distribution network with high penetration of DG units», Measurement: Journal of the International Measurement Confederation, vol. 46, n.o 9, pp. 33193327, 2013.

[55] R. Salim, K. de Oliveira, A. Filomena, M. Resener, y A. Bretas, «Hybrid fault diagnosis scheme implementation for power distribution systems automa- 
tion», IEEE Transactions on Power Delivery, vol. 23, n.o 4, pp. 1846-1856, 2008.

[56] J. Mora, V. Barrera, y G. Carrillo, «Fault Location in Power Distribution Systems Using a Learning Algorithm for Multivariable Data Analysis», IEEE Transactions on Power Delivery, vol. 22, n.o 3, pp. 1715-1721, 2007.

[57] D. Gazzana, G. Ferreira, A. Bretas, A. Bettiol, A. Carniato, L. Passos, A. Ferreira, y J. Silva, «An integrated technique for fault location and section identification in distribution systems», Electric Power Systems Research, vol. 115, pp. 65-73, 2014.

[58] R. Dashti y J. Sadeh, «Fault section estimation in power distribution network using impedance-based fault distance calculation and frequency spectrum analysis», IET Generation, Transmission \& Distribution, vol. 8, n.o 8, pp. 1406-1417, 2014.

[59] F. Trindade y W. Freitas, «Low Voltage Zones to Support Fault Location in Distribution Systems With Smart Meters», IEEE Transactions on Smart Grid, vol. pp, n.o 99, pp. 1-10, 2016.

[60] R. Pérez, «Estrategia inteligente como contribución a la exactitud en la localización de fallas en sistemas eléctricos de distribución aéreos con generación distribuida», UNEXPO, 2017.

[61] S. Jamali y V. Talavat, «Accurate fault location method in distribution networks containing distributed generations», Iranian Journal of Electrical and Computer Engineering, vol. 10, n.o 1, pp. 27-33, 2011.

[62] C. Orozco, A. Bretas, R. Chouhy, A. Herrera, y J. Marín, «Active distribution network fault location methodology: A minimum fault reactance and Fibonacci search approach», International Journal of Electrical Power and Energy Systems, vol. 84, pp. 232-241, 2017.

[63] S. Javadian, M. Haghifam, y N. Rezaei, «A fault location and protection scheme for distribution systems in presence of DG using MLP neural networks», 2009 IEEE Power and Energy Society General Meeting, PES '09, pp. 1-8, 2009.

[64] A. Rafinia y J. Moshtagh, «A new approach to fault location in three-phase underground distribution system using combination of wavelet analysis with ANN and FLS», International Journal of Electrical Power and Energy Systems, vol. 55, pp. 261-274, 2014.

[65] S. Brahma y A. Girgis, «Development of adaptive protection scheme for distribution systems with high penetration of distributed generation», 2003 IEEE Power Engineering Society General Meeting, vol. 4, n.o 1, pp. 56-63, 2003.

[66] S. Conti y S. Nicotra, «Procedures for fault location and isolation to solve protection selectivity problems in MV distribution networks with dispersed generation», Electric Power Systems Research, vol. 79, n.o 1, pp. 57-64, 2009.

[67] S. Jamali y V. Talavat, «Dynamic fault location method for distribution networks with distributed generation», Electrical Engineering, vol. 92, n.o 3, pp. 119-127, 2010. 
[68] S. Lotfifard, M. Kezunovic, y M. Mousavi, «Voltage sag data utilization for distribution fault location», IEEE Transactions on Power Delivery, vol. 26, n.o 2, pp. 1239-1246, 2011.

[69] S. Jamali y A. Bahmanyar, «A Fast and Accurate Fault Location Method for Distribution Networks with DG Using Genetic Algorithms», en Smart Grid Conference (SGC), 2015, 2015, pp. 110-114.

\section{Sobre los autores}

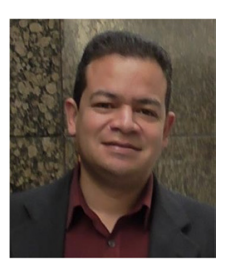

\section{Ramón Enrique Pérez Pineda}

Graduado en el año 2006 como Ingeniero Electricista en la Universidad Nacional Politécnica Antonio José de Sucre (UNEXPO) en Barquisimeto, Venezuela. En los años 2011 y 2018 la UNEXPO le otorga los títulos de Magister Scientiarum en Ingeniería Eléctrica y Doctor en Ciencias de la Ingeniería. Ha sido autor y coautor de varios trabajos de investigación indexados en diferentes bases de datos. Sus principales áreas de investigación son las máquinas eléctricas, sistemas eléctricos de distribución y microredes eléctricas. Actualmente es profesor de la Universidad Politécnica Salesiana en Quito, Ecuador y director de las carreras de Ingeniería Electrónica - Electrónica y Automatización, además de coordinar el Grupo de Investigación en Electrónica, Control y Automatización (GIECA).

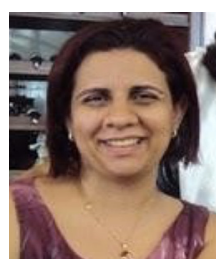

\section{Carmen Luisa Vásquez Stanescu}

De nacionalidad venezolana y rumana. Ingeniero Electricista graduada de la Universidad Nacional Politécnica Antonio José de Sucre (UNEXPO) en 1995. Magister Scientiarum en Ingeniería Eléctrica en 1998 en la misma institución. Doctora en Ciencias Técnicas del Instituto Superior Politécnico "José Antonio Echeverría" de La Habana, Cuba, en el año 2002. Desde 1995 se ha desempeñado como docente e investigadora de la UNEXPO Vicerrectorado Barquisimeto, en las áreas de investigación de Calidad de la Energía Eléctrica, Ingeniería de la Calidad, Transitorios Electromagnéticos y Eficiencia Energética. Adicionalmente, es Coordinadora de la Maestría de Ingeniería Eléctrica, miembro del Comité Académico del Doctorado en Ciencias de la Ingeniería y, desde el año 2014, es Coordinadora de la Unidad Central de Investigación de la UNEXPO. Ha participado en la Acción de Coordinación EFESOS del CYTED (2007-2009) y, actualmente, la Red RITMUS (2018-2021). Ha promovido y participación en 15 proyectos de investigación con financiamiento nacional e internacional, con instituciones AECID, CYTED, Istituto Nazionale Di Fisica Nucleare de Legnaro National Laboratories (Italia), Centro para la Cooperación y para el Desarrollo (CDD) y CITCEA de la Universidad Politécnica de Cataluña (UPC), Barcelona, España, entre otros. Autora del Libro "Eficiencia Energética y calidad de la energía eléctrica. Criterios de diseño, operación y mantenimiento" (2010) y coautora del libro "Eficiencia Energética. Aplicaciones a la 
seguridad del suministro y la sostenibilidad el medio ambiente" (2011). Autora y ponente de 140 trabajos de investigación indexados en diferentes bases de datos.

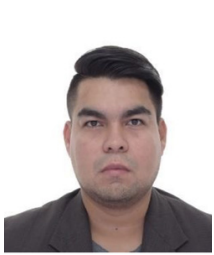

\section{Amelec Jesús Viloria Silva}

Ingeniero Químico con doctorado en Ciencias de la Ingeniería, egresado de la Universidad Nacional Experimental Politécnica Antonio José de Sucre en Barquisimeto, Venezuela. Más de 200 artículos internacionales avalan su actividad investigativa en el área de la estadística y la gestión de productividad. Actualmente es Profesor titular en la Universidad de la Costa, Barranquilla Colombia. 